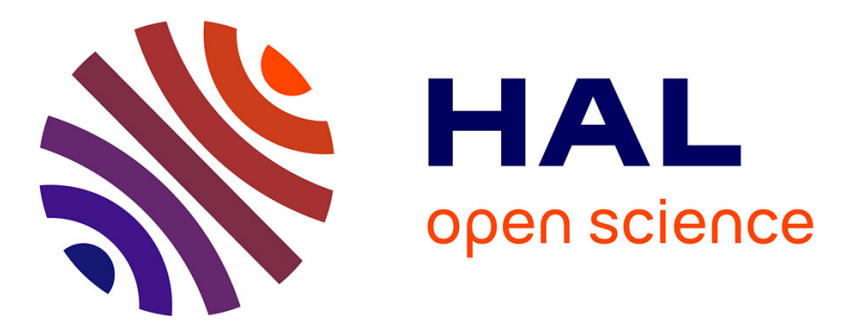

\title{
Wrinkle development analysis in thin sail-like structures using MITC shell finite elements
}

Daniele Trimarchi, Marina Vidrascu, Dominic Taunton, Stephen R. Turnock, Dominique Chapelle

\section{- To cite this version:}

Daniele Trimarchi, Marina Vidrascu, Dominic Taunton, Stephen R. Turnock, Dominique Chapelle. Wrinkle development analysis in thin sail-like structures using MITC shell finite elements. Finite Elements in Analysis and Design, 2013, 64, pp.48-64. 10.1016/j.finel.2012.09.005 . hal-00733994

\section{HAL Id: hal-00733994 \\ https://hal.inria.fr/hal-00733994}

Submitted on 20 Sep 2012

HAL is a multi-disciplinary open access archive for the deposit and dissemination of scientific research documents, whether they are published or not. The documents may come from teaching and research institutions in France or abroad, or from public or private research centers.
L'archive ouverte pluridisciplinaire HAL, est destinée au dépôt et à la diffusion de documents scientifiques de niveau recherche, publiés ou non, émanant des établissements d'enseignement et de recherche français ou étrangers, des laboratoires publics ou privés. 


\title{
Wrinkle development analysis in thin sail-like structures using MITC shell finite elements
}

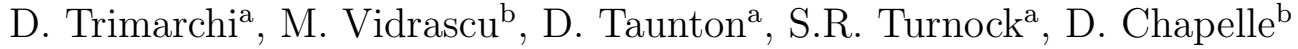 \\ ${ }^{a}$ Fluid Structure Interactions Research Group, University of Southampton, U.K. \\ ${ }^{b}$ Inria, Macs Team, France
}

\begin{abstract}
We propose a method of modelling sail type structures which captures the wrinkling behaviour of such structures. The method is validated through experimental and analytical test cases, particularly in terms of wrinkling prediction. An enhanced wrinkling index is proposed as a valuable measure characterizing the global wrinkling development on the deformed structure. The method is based on a pseudo-dynamic finite element procedure involving non-linear MITC shell elements. The major advantage compared to membrane models generally used for this type of analysis is that no ad hoc wrinkling model is required to control the stability of the structure. We demonstrate our approach to analyse the behaviour of various structures with spherical and cylindrical shapes, characteristic of downwind sails over a rather wide range of shape and constitutive parameters. In all cases convergence is reached and the overall flying shape is most adequately represented, which shows that our approach is a most valuable alternative to standard techniques to provide deeper insight into the physical behaviour. Limitations appear only in some very special instances in which local wrinklingrelated instabilities are extremely high and would require specific additional treatments, out of the scope of the present study.
\end{abstract}

Keywords: Sail Modeling, MITC Shells, Wrinkling, Wrinkling Index

\section{Introduction}

Sail-like thin laminates, and more generally inflatable structures, have been analysed with Finite Element (FE) methods, see e.g. [8, 9]. In such studies the fabric has generally been modelled using the membrane model, which assumes that the bending components are negligible. 
The constant strain triangle (CST) membrane element has often been adopted for inflatable structures [18, 23, 2] or sail-type analysis $[9,22,16]$, for which the ratio $t / L$ - where $t$ is the fabric thickness and $L$ a characteristic size - is very small. The success and longevity of this model are partly due to its simplicity; the CST membrane is not without problems, however, and examples can be found where this class of model produces incorrect answers [20].

More complete iso-parametric models have been adopted for general inflatable structure analysis $[3,6]$. There are, however, particular combinations of load and boundary conditions for which the membrane problem is ill-posed. When this happens, for example in point-supported structures such as downwind sails (see Figure 1), the membrane model itself is inadequate to represent the structural deformation, as the state of the structure is bending-dominated, or of mixed membrane-bending type.
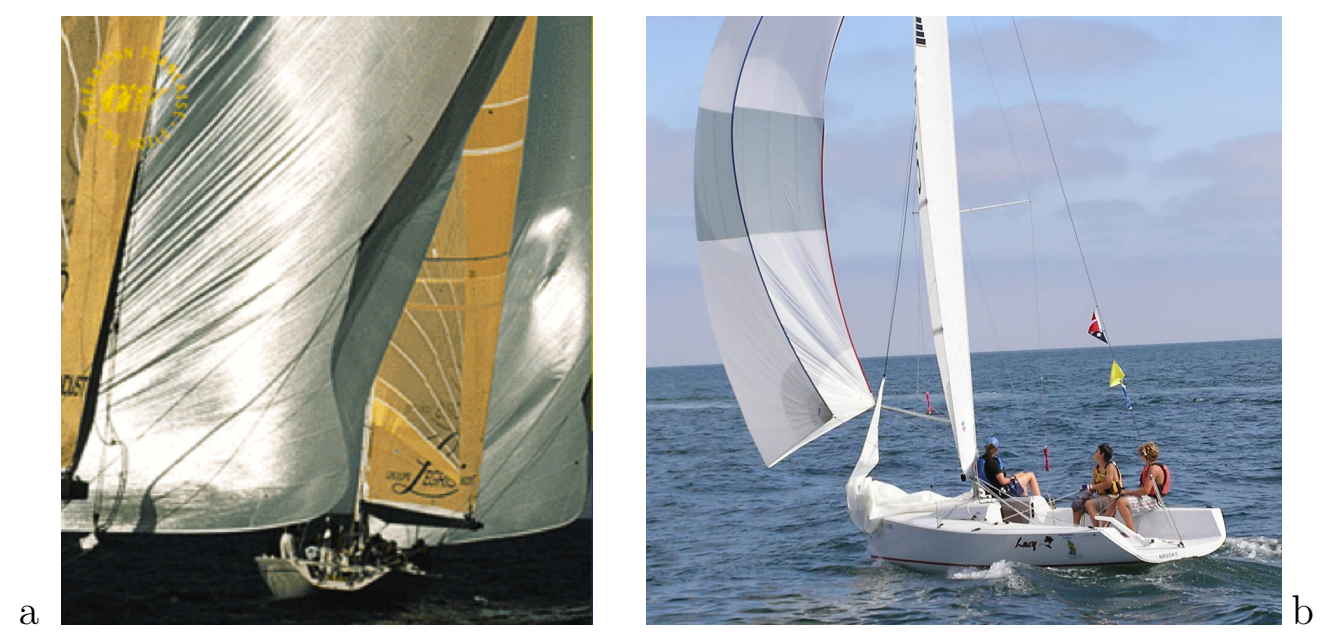

Figure 1: Examples of wrinkled downwind sails - a: Courtesy of Christian Février and $b$ : Courtesy of Bravura Yachts

In such situations a series of oscillations arise on the deformed fabric surface, which is then said to be "wrinkled", as in Figure 1. The development of such oscillations is buckling related, thus mostly controlled by the bending stiffness. The membrane model being unable to represent such configurations, singularities appear and convergence cannot be reached. Generally, these difficulties are overcome using ad hoc wrinkling models [11, 15], and applications examples for sail deformation analysis can be found in [10, 21]. 
Similar behaviour can be identified also along the free edges of the sail where the membrane stiffness is ineffective [7] - and such border singularities are often avoided by introducing cable or beam elements as supporting frames [12].

An alternative approach is proposed here using a finite element shell model. It is then possible to numerically reproduce the wrinkling development. The suitability of using shell finite elements for planar physical membranes has been shown by $[29,28]$. In the present paper, however, we analyse a fully curved geometry. The intrinsic ill-posedness of the problem induced by the above-mentioned physical instabilities, deriving from the very small thickness of the fabric, is overcome with the use of Newmark-type dynamic schemes with Rayleigh damping, see e.g. [3]. Our work is thus concerned with applying and assessing this type of procedure when used with effective shell elements to model sails undergoing wrinkling. Some specific geometries were selected as representative of downwind sail shapes. These structures were then loaded with constant pressure, the value of which lies in the typical range for downwind yacht sails. Although complex interactions exist between the deformed shape - including the wrinkles - and the distribution of the actual wind loading [26], the work presented here focuses on the structural response and provides therefore a basis for the coupled FSI analysis [19, 27, 25].

The aim of this work is then to computationally characterize the nature and the behaviour of the wrinkling development with a particular concern for downwind sail configurations. Section 2 describes the method adopted for the analysis of thin wrinkled shells. In Section 3 this method is assessed based on experimental results and analytical solutions published in the literature [29], for a flat membrane with prescribed boundary displacements. The nature of wrinkling is then discussed in terms of stability in Section 4, by an eigenvalue analysis of the tangent stiffness matrix of the structure. The next sections are devoted to specific applications of the proposed procedure to sail configurations, with detailed sensitivity studies with respect to various parameters characterizing discretization, geometry and material properties. In Section 5, mesh sensitivity is examined with particular regard to wrinkling generation. Section 6 then presents a parametric analysis for a spherical geometry to investigate the influence of fabric thickness, Young's modulus, and the applied load on the wrinkle wavelength and the number of wrinkles. Then Section 7 examines the effect of shape variations in Gennakerand Spinnaker-type geometries. Finally, we give some concluding remarks in 
Section 9.

\section{Methods}

In this Section we outline the major methodological concepts used in our analysis of wrinkled shells.

\subsection{Shell kinematical assumptions and finite element approximation}

Non-linear iso-parametric four-node shell elements of the MITC family (Mixed Interpolation Tensorial Components) have been used for the finite element analysis. The detailed formulation of MITC shell elements can be found in [3] and in [7], where detailed considerations on the shell physical behaviour are also presented. For completeness, however, we will provide a summary of the kinematical assumptions and of the finite element discretizations considered.

The underlying mathematical shell model is based on the so-called Reissner-Mindlin kinematical assumption, stating that any material line initially orthogonal to the shell midsurface in the undeformed configuration remains straight and unstretched in all deformed states. Note that we do not assume further - as in the Kirchhoff-Love kinematical assumption - that the deformed material line remains orthogonal to the deformed midsurface, see e.g. $[5,17]$ and references therein for shell elements based on the KirchhoffLove assumption. We thus allow for all types of deformation - including transverse shear - in the material.

The specific shell element considered - namely, the MITC4 element - was selected for its remarkable effectiveness, robustness with respect to various numerical pathologies such as locking affecting shell analysis, and versatility, as demonstrated in $[4,7]$. In particular, this element has been shown to accurately represent both membrane- and bending-dominated types of behaviour, and to be also effective for extremely thin structures, hence removing the need for switching between so-called "thick and thin" shell elements depending on the application considered.

Denoting the local coordinates by $(r, s, z)$ varying between -1 and +1 inside an element, the Euclidean position vector of a point within this element for a configuration indexed by $\ell$ is given by

$$
{ }^{\ell} \vec{X}=\sum_{k=1}^{4} \lambda_{k}(r, s)\left({ }^{\ell} \vec{X}^{(k)}+z \frac{t^{(k)}}{2}{ }^{\ell} \vec{V}_{n}^{(k)}\right)
$$


where ${ }^{\ell} \vec{X}^{(k)},{ }^{\ell} \vec{V}_{n}^{(k)}$ and $t^{(k)}$ respectively denote the position vector, unit transverse direction vector ${ }^{1}$, and thickness parameter at node $k$ in configuration $\ell$, while $\lambda_{k}$ is the associated finite element shape function. Here, since we have four-node elements, standard bilinear interpolation functions are used. Then, based on the iso-parametric construction discrete displacements within a total Lagrangian formulation are obtained by subtracting the initial position, viz.

$$
{ }^{\ell} \vec{U}=\sum_{k=1}^{4} \lambda_{k}(r, s)\left({ }^{\ell} \vec{U}^{(k)}+z \frac{t^{(k)}}{2}\left({ }^{\ell} \vec{V}_{n}^{(k)}-{ }^{0} \vec{V}_{n}^{(k)}\right)\right),
$$

where ${ }^{\ell} \vec{U}^{(k)}$ then denotes nodal displacements with respect to the initial configuration. Note that, under a small displacement assumption - which we do not use in our modelling - the difference between transverse direction vectors could be parametrized in the standard form

$$
{ }^{\ell} \vec{V}_{n}^{(k)}-{ }^{0} \vec{V}_{n}^{(k)}=-\alpha^{(k) 0} \vec{V}_{2}^{(k)}+\beta^{(k) 0} \vec{V}_{1}^{(k)},
$$

where $\left({ }^{0} \vec{V}_{1}^{(k)},{ }^{0} \vec{V}_{2}^{(k)},{ }^{0} \vec{V}_{n}^{(k)}\right)$ defines an orthonormal basis in the reference configuration - hence, $\left({ }^{0} \vec{V}_{1}^{(k)},{ }^{0} \vec{V}_{2}^{(k)}\right)$ are unit vectors lying in the tangential plane to the midsurface - and $\left(\alpha^{(k)}, \beta^{(k)}\right)$ denote associated rotation angles. In our case, we consider large displacements and this parametrization by rotation angles is only used in the linearized increments needed in the Newton-type algorithm [3].

These kinematical assumptions are then incorporated in a $3 \mathrm{D}$ variational formulation with a plane stress assumption to obtain the discrete (finite element) problem. We emphasize that, in MITC elements, special care is exercised to circumvent specific difficulties associated with thin structures [7]. In particular, various forms of numerical locking must be avoided while preserving the stability in the energy. To that purpose, in MITC elements specific interpolation rules for the various components of the strains are defined based on well-chosen "tying-points". Some other shell finite element procedures instead rely on reduced integration, but specific controls are required in order to avoid the zero energy modes called hourglass modes [30]. In our computations we used the MITCNL software developed at Inria, an implementation of the non-linear MITC shell elements.

\footnotetext{
${ }^{1}$ The transverse direction vector is not normal to the midsurface in general deformed configurations
} 


\subsection{Pseudo-dynamical scheme}

The finite element analysis of thin structures such as sail type laminates is likely to encounter convergence issues. For such materials, typical aspect ratios - namely, thickness over characteristic size - varies between $10^{-4}$ and $10^{-3}$. Since wrinkling modes are essentially controlled by the bending stiffness - proportional to the cube of the aspect ratio [7]- this leads to configurations where very little stability prevails, and even some intermediate stages - e.g. in incremental loading - where no stable static solution exists. Various techniques can be adopted in order to overcome such instabilities, and we here choose to employ a pseudo-dynamic procedure with artificial damping. Namely, we compute a static solution by following a dynamical path in which we use the natural modelling of the inertia and stiffness effects, and incorporate some Rayleigh damping defined in an ad hoc manner. Of course, the time variable in this procedure is then a "pseudo-time", which should not be considered as the physical time. The dynamic equation solved is then of the type (see e.g. [3])

$$
\mathbf{M} \ddot{\mathbf{Y}}+\mathbf{C} \dot{\mathbf{Y}}+\mathbf{K}(\mathbf{Y})=\mathbf{F}(t)
$$

where $\mathbf{Y}$ denotes the vector of displacement degrees of freedom, $\mathbf{F}$ the load vector, $\mathbf{M}$ the usual (consistent) mass matrix, $\mathbf{K}(\mathbf{Y})$ the non-linear stiffness matrix-operator, and

$$
\mathbf{C}=c_{1} \mathbf{M}+c_{2} \mathbf{K}_{0},
$$

with $c_{1}, c_{2}$ constant values and $\mathbf{K}_{0}$ a reference stiffness matrix to be specified - e.g., the tangent stiffness matrix in the undeformed configuration. For a linear behaviour with $\mathbf{K}(\mathbf{Y})=\mathbf{K}_{0} \mathbf{Y}$ we can use modal decomposition based on the eigenmodes of $\mathbf{K}_{0}$ with respect to $\mathbf{M}$, and the damping ratio for a given natural frequency $\omega$ then gives

$$
\xi=\frac{c_{1}+c_{2} \omega^{2}}{2 \omega}
$$

For a linear harmonic oscillator, tuning this coefficient has the effect of modifying the damping characteristics of the system. No damping $(\xi=0)$ results in infinite oscillations, while $0<\xi \leq 1$ leads to oscillations damped with a characteristic time constant of $1 /(\xi \omega)$, until no oscillation is encountered for $\xi=1$ (so-called "critical damping"). For $\xi>1$ the system is overdamped - i.e. takes a longer time to stabilize than for $\xi=1$ - and for $\xi \gg 1$ the corresponding characteristic time is $2 \xi / \omega$. When applying Rayleigh damping, 
a linear structure would then behave as a set of such harmonic oscillators. Since the damping ratio is a function of the modal frequency - recall (5) - every eigenmode is then damped with a different time constant, where the fastest convergence is obtained for the critical value $\xi=1$. This implies that no given eigenmode of eigenfrequency $\omega_{i}$ can be damped any faster than with the time constant $1 / \omega_{i}$. Hence, a natural strategy consists in adjusting $c_{1}$ and $c_{2}$ so that the fundamental eigenmode is critically damped $\left(\xi\left(\omega_{1}\right)=1\right)$, and in order to control the damping time constants of higher frequency modes based on the characteristic time $2 \xi\left(\omega_{i}\right) / \omega_{i}$. This leads to

$$
c_{1}=\omega_{1}, \quad c_{2}=1 / \omega_{1},
$$

corresponding to a global time constant of $1 / \omega_{1}$. Of course, this simplified reasoning holds for a linear structure, and in our case it is only approximate, although it can be applied to small oscillations around a given configuration, when using the tangent stiffness operator.

Regarding the time discretization strategy, we use a mid-point rule, namely,

$$
\left\{\begin{array}{l}
\ddot{\mathbf{Y}}_{n+\frac{1}{2}}=\frac{\dot{\mathbf{Y}}_{n+1}-\dot{\mathbf{Y}}_{n}}{\Delta t} \\
\dot{\mathbf{Y}}_{n+\frac{1}{2}}=\frac{\dot{\mathbf{Y}}_{n+1}+\dot{\mathbf{Y}}_{n}}{2}=\frac{\mathbf{Y}_{n+1}-\mathbf{Y}_{n}}{\Delta t}=\frac{2\left(\mathbf{Y}_{n+\frac{1}{2}}-\mathbf{Y}_{n}\right)}{\Delta t}
\end{array}\right.
$$

which we then substitute in the dynamical equation (3) written at time " $n+$ $\frac{1}{2}$ ". This non-linear implicit equation is solved based on a Newton-Raphson algorithm, in which we also allow for time adaptation when convergence difficulties are encountered in the Newton loop. Typically, at the beginning of the solution process the physical instability and the rapidly-changing stiffness operator tend to produce oscillations, which can only be handled with small time-steps. As the computation proceeds the time step can be progressively increased, until it ideally takes infinite values for a steady equilibrium state. The use of a variable time-step is thus necessary both for handling stability issues and for reducing the computation time.

The resulting global solution algorithm is then summarized in Figure 2.

\subsection{Measures of wrinkling}

Two main measures are generally used in order to characterize surface wrinkling: the number $n_{w}$ - or wavelength $L_{w}$ - of the wrinkles appearing on the deformed surface and their amplitude $A_{w}[29,28]$. A third measure is 


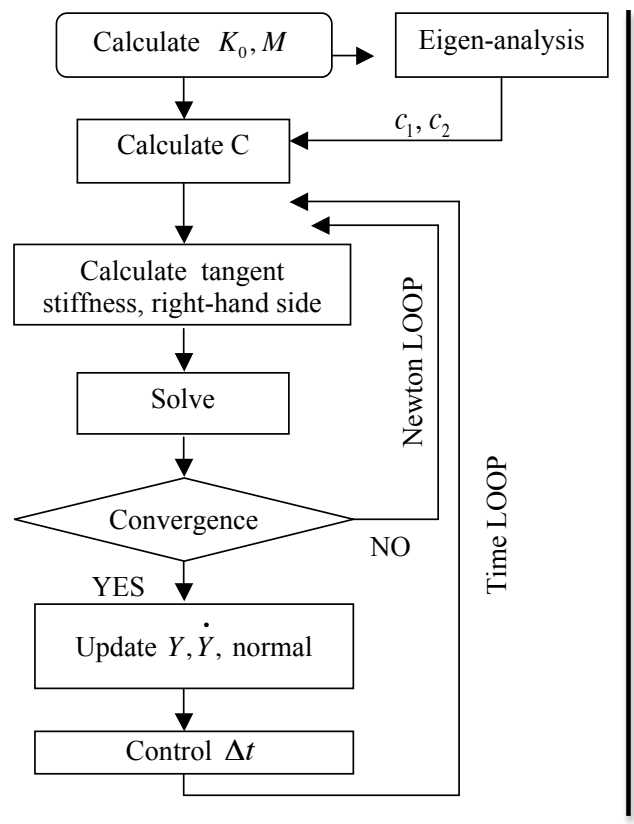

Control $\Delta t$ :

- if Newton converges in less than $\mathrm{N}$ iterations for $\mathrm{M}$ successive time-steps: $\Delta t=2 \Delta t$

- if Newton does not converge: re-initialize to previous time-step $\Delta t=\Delta t / 2$

Figure 2: Flow chart summarizing the analysis: eigen-analysis, non-linear finite element solution and time-step adaptation

here introduced, which describes the global state of a wrinkled section. This is defined as $I_{w}=n_{w} \cdot \sigma$, where $\sigma$ is the standard deviation of the section from a smooth regression curve fitting the wrinkled cross-section. This index thus takes similar values for a large number of small wrinkles or a small number of large wrinkles and of course for a smooth surface the index vanishes.

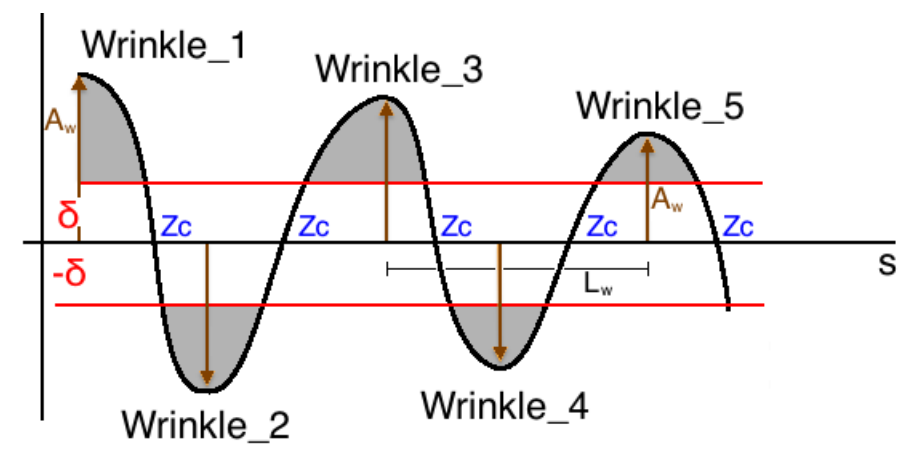

Figure 3: Wrinkling recognition criterion 
Wrinkling measures were computed with an automatic wrinkling recognition algorithm for planar cross-sections. This algorithm uses a regression of the cross-section with a polynomial of order five, and a re-parametrization with the arc length of the polynomial curve. Consequently, we simply compare the transformed cross-section to the straight arc-length axis as visualized in Figure 3. Calculating the standard deviation $\sigma$, the number of wrinkles is determined by following the arc-length and counting a new wrinkle every time the curve intersects the thresholds $-\delta$ or $+\delta$, where

$$
\delta=\max \left(\alpha \sigma, \sigma_{M I N}\right),
$$

directly after a zero crossing $Z_{c}$, for a well-chosen factor $\alpha$. The minimum height of the threshold $\sigma_{M I N}$ is calculated as a percentage of the section length, and avoids taking into account excessively small oscillations, which e.g. may be induced by the finite element discretization. In practice, suitable values are $\alpha=0.2, \sigma_{M I N}=s_{M A X} / 1000$, where $s_{M A X}$ is the maximum coordinate of the arc-length.

\section{Validation test case}

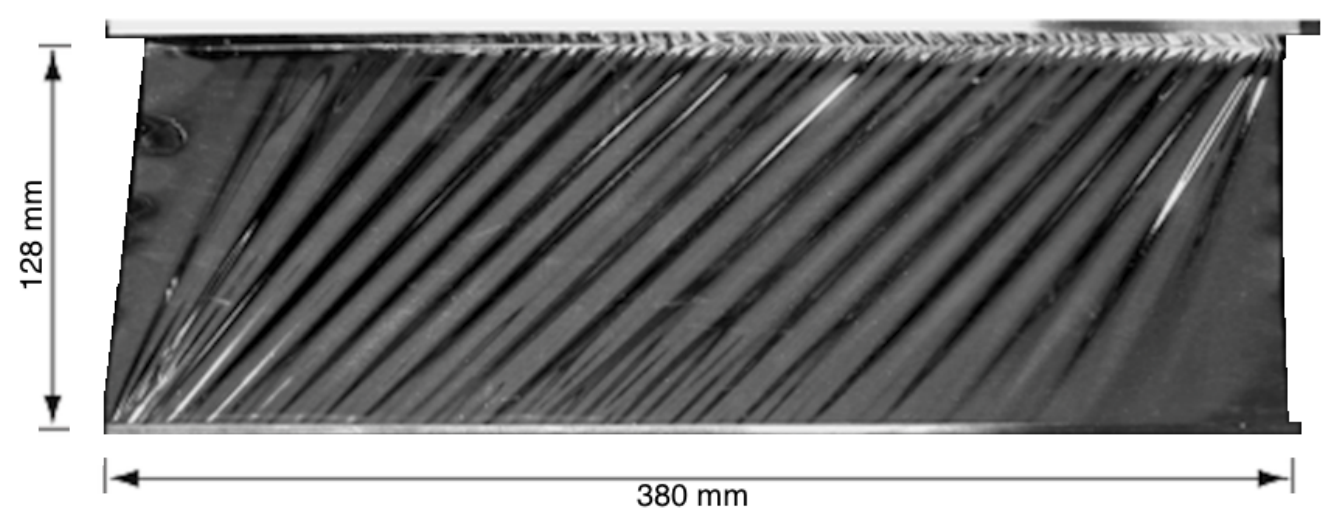

Figure 4: Snapshot of membrane in shear (taken from [29], Fig. 3)

Wrinkle evaluations were proposed by $[24,28,29]$. In this paper we have focused on the work of [29], which analysed an initially planar rectangular membrane with prescribed shearing displacements on one side, as shown in Figure 4. The membrane was made of a thin film of Kapton ${ }^{\circledR}$, the physical quantities of which are reported in Table 1. We point out that the aspect 
Table 1: Kapton ${ }^{\circledR}$ physical quantities reported by [29]

\begin{tabular}{|c|c|c|c|c|}
\hline$t$ & $E$ & $\nu$ & $L$ & $H$ \\
\hline$[\mathrm{mm}]$ & {$\left[\mathrm{N} / \mathrm{mm}^{2}\right]$} & {$[-]$} & {$[\mathrm{mm}]$} & {$[\mathrm{mm}]$} \\
\hline 0.025 & 3500 & 0.31 & 380 & 128 \\
\hline
\end{tabular}

ratio of this particular Kapton ${ }^{\circledR}$ membrane $\left(t / L=1.9510^{-4}\right)$ lies in the same range as the material used for downwind sail production.
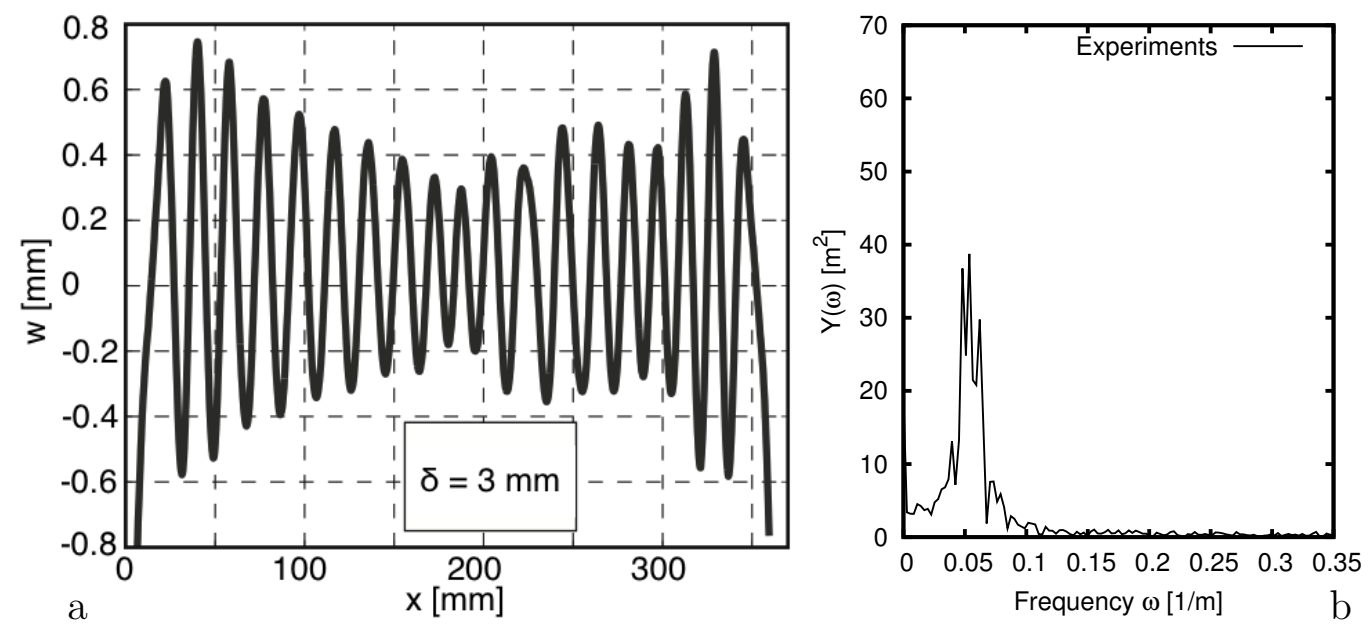

Figure 5: Experimental results - $a$ : Measured deformed section with $3 \mathrm{~mm}$ imposed displacement (reproduced from [29], Fig. 4) and $b$ : FFT analysis of the deflection

In this configuration the membrane is in a shear-dominated mode, hence it is likely to produce several wrinkles oriented at $45^{\circ}$. It was reported that, although the imposed displacement was increased gradually and monotonically, changes in the number of wrinkles occur suddenly, as the effect of an instability originating near the edge. The number, amplitude and wavelength of such oscillations is dependent upon the material and the magnitude of the prescribed displacement. The measured deformed central cross-section of the membrane is plotted in Figure 5. A discrepancy should however be underlined: the declared length of the membrane is $380 \mathrm{~mm}$, whereas the graph maximum $x$-value in Figure 5 is only $\approx 360 \mathrm{~mm}$. We should also note that the symmetry properties expected from the loading considered are not entirely obtained in the response. In particular, a rather surprising irregularity in 
the amplitude of the oscillations is observed near the center for $x>180 \mathrm{~mm}$. We conjecture that this is due to a slip in the displacement prescription, as is clearly noticeable in Figure 4 (right-hand side of top edge).

An initial out-of-plane displacement map - defined as a linear combination of eigenmodes - was applied on the undeformed structure for the numerical results published in [29]. However, the influence of this map was negligible in the final result, and the need for this initialization procedure is probably due to the use of the static solver implemented in the Abaqus commercial code.

We reproduced this experiment with our proposed method. Lateral sides were left free, whereas all degrees of freedom of the bottom edge were fixed, and displacements were prescribed on the top edge. No initial out-of-plane mapping or pre-stress was needed. Due to the expected 45-degrees shear mode, we directly considered adapted meshes aligned with this privileged direction, see Figure $7 a$. The pseudo-dynamic algorithm was able to capture the final deformed shape. However, of course since no initial imperfections were applied, out-of-plane oscillations only appear when the top edge displacements reach a given finite value.

\subsection{Mesh sensitivity for the validation test case}

Table 2: MITC4 results for series of meshes considered

\begin{tabular}{|c|c|c|c|c|c|}
\hline Subdivisions & Elements & $n_{w}$ & $W_{\text {centre-line }}[\mathrm{mm}]$ & $\sigma$ & $I_{w}$ \\
\hline $65 \times 50$ & 3038 & 13 & 0.47 & 0.250 & 3.25 \\
\hline $105 \times 50$ & 4998 & 15 & 0.47 & 0.362 & 5.43 \\
\hline $160 \times 50$ & 7693 & 19 & 0.40 & 0.232 & 4.41 \\
\hline $210 \times 50$ & 10143 & 20 & 0.38 & 0.178 & 3.56 \\
\hline $420 \times 75$ & 28638 & 19 & 0.40 & 0.136 & 2.58 \\
\hline $420 \times 100$ & 42183 & 21 & 0.35 & 0.155 & 3.23 \\
\hline $630 \times 150$ & 55803 & 20 & 0.37 & 0.140 & 2.8 \\
\hline $880 \times 150$ & 130673 & 20 & 0.37 & 0.140 & 2.8 \\
\hline
\end{tabular}

Mesh sensitivity was explored for eight meshes, the characteristics of which are reported in Table 2. Figure $6 a$ shows the mesh sensitivity in terms of number $n_{w}$ and the proposed wrinkling index $I_{w}$ defined in Section 2.3. Figure $6 b$ reports the mesh sensitivity in terms of the amplitude of wrinkles 

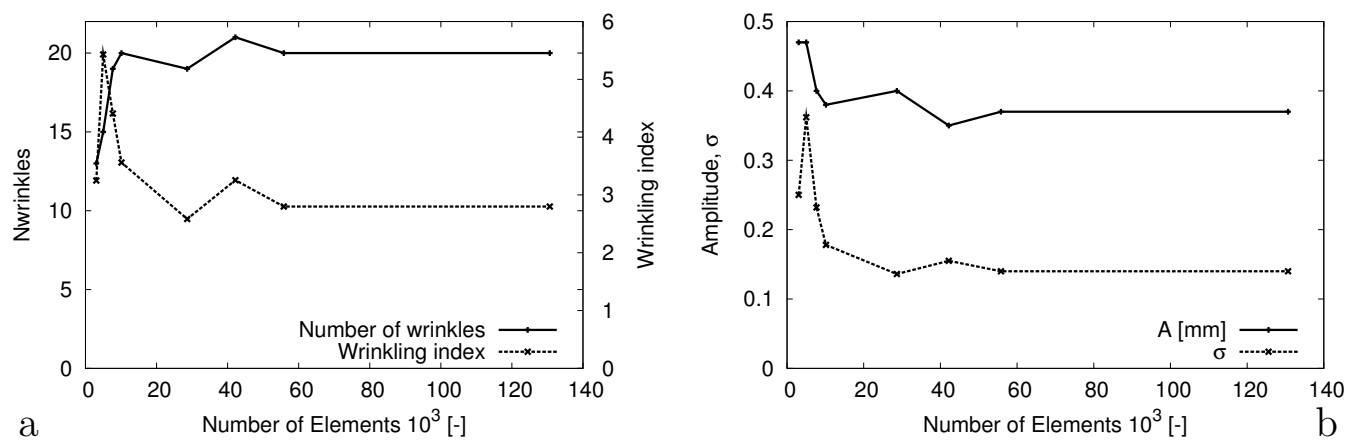

Figure 6: Mesh sensitivity for the validation test case $-a$ : Number of wrinkles $n_{w}$ and wrinkling index $I_{w} ; b$ : Amplitude $A_{w}$ and standard deviation $\sigma$

$A_{w}$ and the standard deviation $\sigma$, defined with respect to the undeformed straight configuration of the center cross-section.

A significant mesh sensitivity is experienced. This is not surprising since several elements are needed in order to reproduce every wrinkle, the wavelength of which may be very small, especially along the constrained sides. As a general rule of thumb, the mesh should exceed 20 elements per wrinkle in order to capture the correct structural behaviour. When the mesh is fine enough the results converge to the solution; this is confirmed by the good degree of accuracy achieved in the comparison with the experiments (see Section 3.2).

\subsection{Comparison with experimental results}

The results discussed here are those obtained with a converged mesh (630x150 elements). The general behaviour is well-captured in terms of wrinkle shape, size and orientation. Fine details are also well-represented, such as the smaller oscillations observed in between two large wrinkles near the top and bottom edges, see Figure 4. Compared to the experimental results, however, our numerical solutions feature a more regular response and the predicted number of wrinkles (20) exceeds the experimental value (19).

Figure 8 compares the experimental and the numerical cross-sections. Since the experimental curve is of non-zero mean, an appropriate shift - of specific value $(-7,0.8)[\mathrm{mm}]$ - was applied to make the curves comparable.

This comparison shows a good accuracy in reproducing the experimental behaviour. The response is particularly accurate both in terms of amplitude and wave-length. Five zones can be identified, as illustrated in Figure 8. 


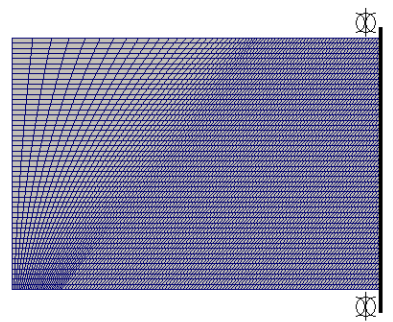

a

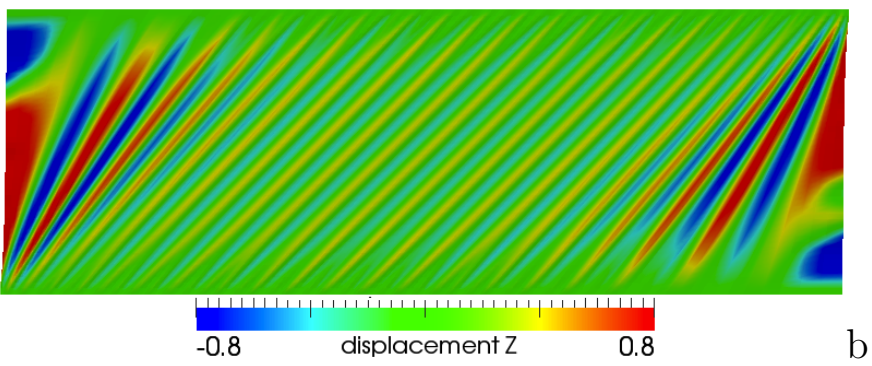

$\mathrm{b}$

Figure 7: $a$ : Adapted mesh with 210x50 elements (symmetry); $b$ : Deformed shape for $3 \mathrm{~mm}$ displacement
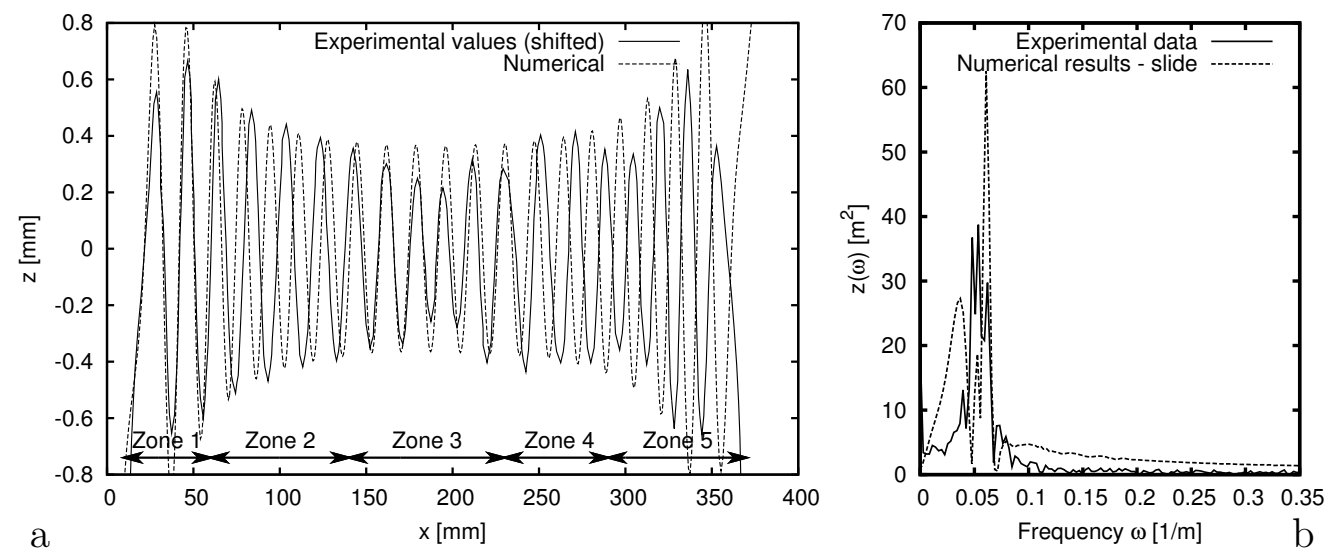

Figure 8: Numerical/experimental comparison for 630x150 mesh - $a$ : Deformed crosssections; $b$ : FFT of deflections

In Zone 1 the three "boundary" wrinkles are well-captured. A transition region (Zone 2) is then seen where the amplitude is well-captured, but five wrinkles are found instead of four. In the centre of the membrane (Zone 3 ), the solution is accurate in terms of wave-length. The amplitude is less accurate, but this could be due to possible imperfections in the experimental setup, as already mentioned, or in the material - constitutive and geometric - properties. Zones 4 and 5 display anti-symmetry - with respect to Zones 2 and 1 , respectively - in the numerical solution, compared to which a phase inversion is seen in the experimental curve for $x>250 \mathrm{~mm}$. This further justifies investigating material and boundary conditions effects, as follows.

The effect of the material was then investigated with a new analysis, where the fabric thickness was increased by $10 \%$. The results are displayed in 


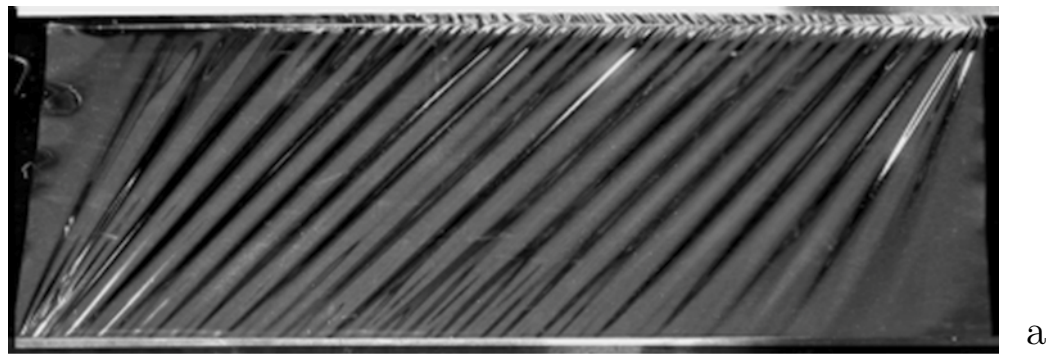

a

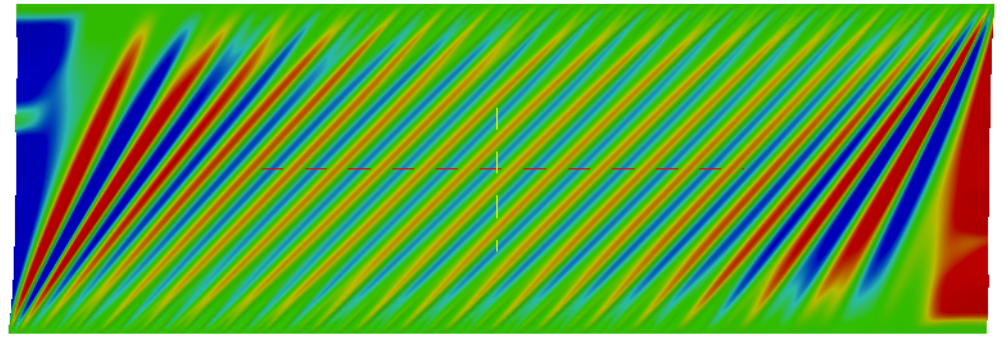

$\mathrm{b}$

Figure 9: Comparison of (a) experimental and (b) numerical deformed shapes
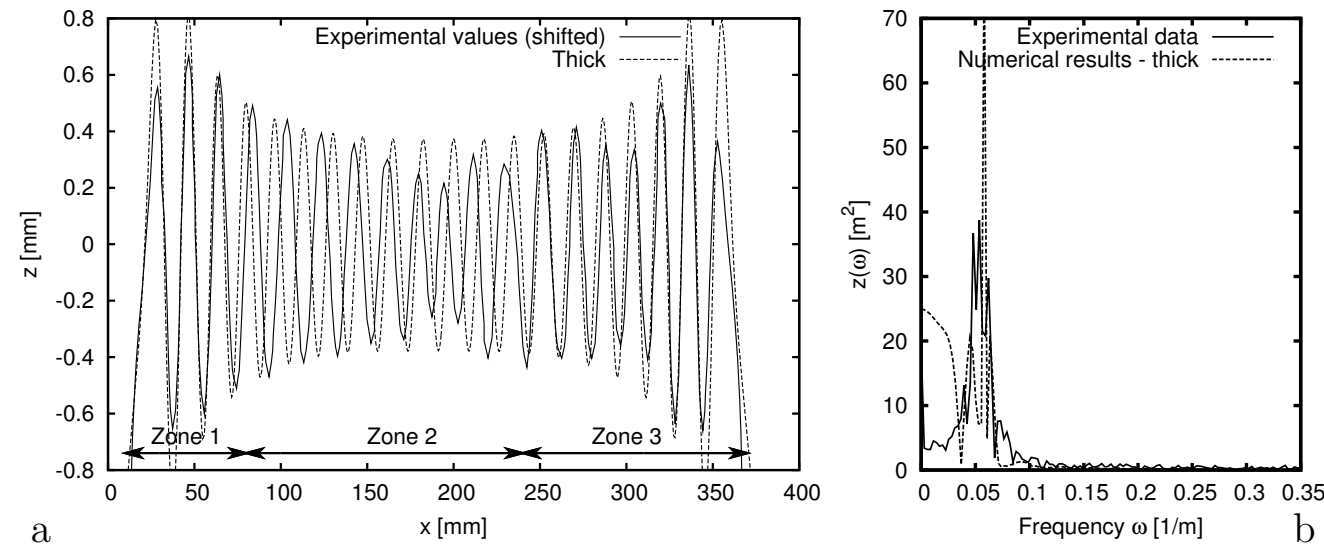

Figure 10: Effect of $10 \%$ thickness increase on the deformed shape of the membrane in shear - $a$ : Numerical/experimental comparison; $b$ : FFT of the deflections

Figure 10. We now have a good representation of the experimental behaviour in the two lateral parts - see new Zones 1 and 3 in this Figure - whereas the numerical wave-length of the wrinkles is still higher - although lower than for the thinner membrane - in the central Zone 2. This is also confirmed by the comparison of the Fourier transforms of the experimental and numerical solutions in Figure 8 and Figure 10, which shows a much narrower frequency 
content in the numerical solutions, with a peak closer to the center of the experimental frequency range in the thick case.
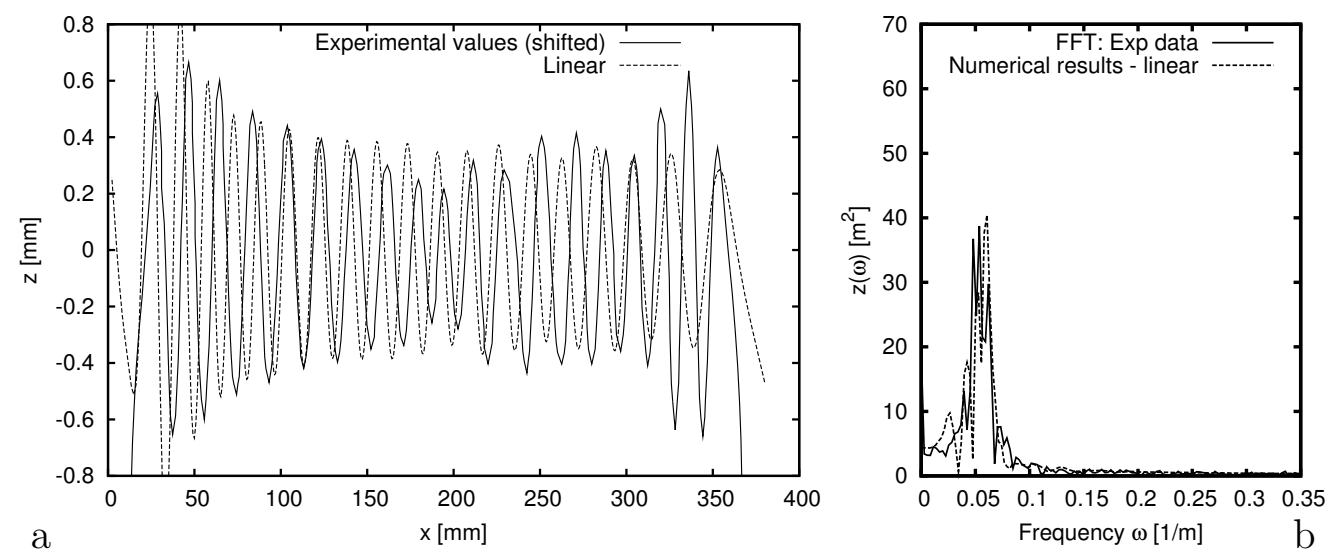

Figure 11: Effect of the linear constraint on the deformed shape of the membrane in shear - left: Numerical/experimental comparison; $b$ : FFT of the deflections

The influence of the boundary condition was then analysed by prescribing a lateral displacement varying linearly from the value $\delta$ at the top-left corner to zero at the top-right corner, in order to model a slip in the boundary condition. The resulting wrinkles are shown in Figure 11, where we observe a less regular behaviour in the numerical solution starting around the middle of the cross-section, as for the experimental curve, although the behaviour near the right side is less accurate. Note also in Figure 11 that the Fourier transform of the numerical and experimental solutions are now very similar.

Finally, it appears that our numerical procedure adequately captures the experimental behaviour of [29], taking into account the various experimental uncertainties.

\subsection{Comparison with analytical estimates and numerical results}

In [29] some analytical derivations are performed for the case considered, under various simplifying assumptions. In particular, the wrinkles half wavelength and amplitude are respectively estimated as

$$
\lambda_{\mathrm{an}}=\sqrt{\frac{\pi H t}{3\left(1-\nu^{2}\right) \gamma}}, \quad A_{\mathrm{an}}=\sqrt{\frac{2 H t}{\pi} \cdot \sqrt{\frac{(1-\nu) \gamma}{3(1+\nu)}}},
$$


with $H$ and $L$ the height and width of the sheet, $\gamma=\delta / H$ the shear deformation. Substituting the above-given numerical values we find $\lambda_{\text {an }}=14.4 \mathrm{~mm}$ and $A_{\mathrm{an}}=0.36 \mathrm{~mm}$. In our numerical solution we have $\lambda \approx 16 \mathrm{~mm}$ and $A \approx 0.35 \mathrm{~mm}$ when measuring near the center, which is in good agreement with the analytical values.

Numerical experiments were also performed in [29] using Abaqus. Three regular meshes were tested, using so-called S4R5 shell four-node elements based on an approximate enforcement of the Kirchhoff-Love kinematical assumption, see Abaqus manual [1] for more detail. The first mesh element size was set slightly smaller than the wrinkle half-wavelength. The finer meshes were obtained by increasing the number of elements by factors two and four, approximately. Figure 12 visualizes the resulting deformed solution, and we also notice in this Figure a peculiarity in the prescribed boundary conditions, namely, that only translations along the $x$-direction were allowed on the two lateral sides. It is argued in [29] that these additional constraints may induce two extra oscillations, which are then subtracted from the counted number of wrinkles. In order to recall the existence of these extra oscillations we explicitly list the values of [29] as " $n+2$ " in Table 3 . We see that our converged values - recall Table 2 - are intermediate between the converged values of Table 3 with and without the additional two wrinkles.

We further note that the smaller oscillations observed in the experimental results near the top and bottom edges are much more adequately captured in our numerical solutions than in the Abaqus numerical results given in [29].

Table 3: Abaqus results for membrane in shear (taken from [29], Table 4)

\begin{tabular}{|c|c|}
\hline N. elements & $N_{\text {wrinkles }}$ Abaqus \\
\hline 3960 & $16+2$ \\
\hline 6950 & $19+2$ \\
\hline 13134 & $19+2$ \\
\hline
\end{tabular}

\section{Wrinkles and equilibrium}

As wrinkling is a form of buckling, its development can be analysed in terms of stability. An equilibrium configuration is called stable when after a small disturbance the system returns to its initial configuration. An 


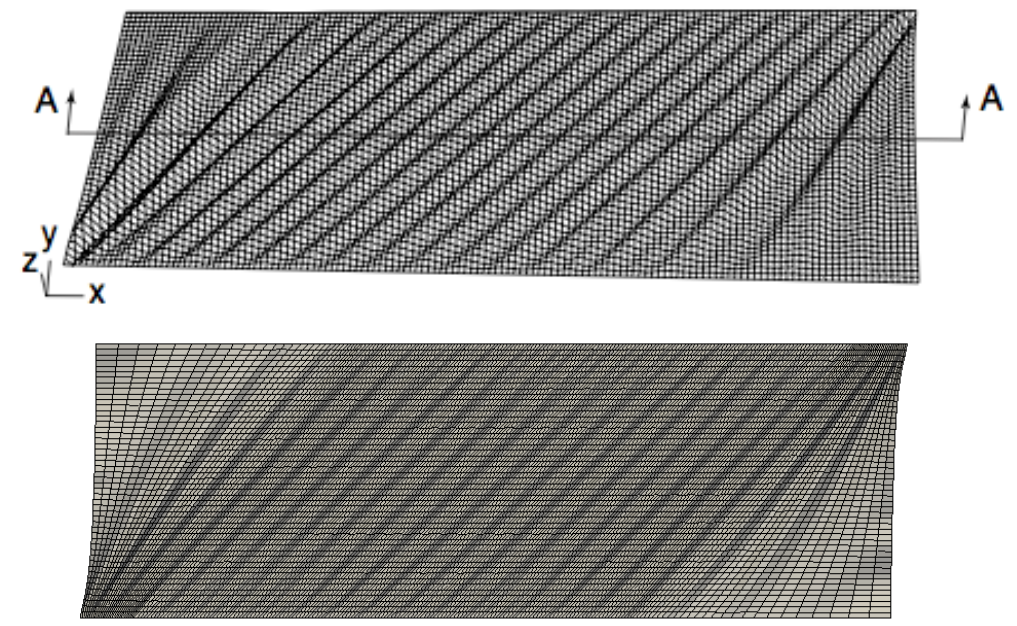

Figure 12: Top: Perspective view of the wrinkle pattern for the Abaqus result (taken from [29], Fig.6); Bottom: Wrinkle pattern for the MITC4 result (real displacements magnified by factor 2.5 )

indifferent equilibrium configuration is encountered when any perturbed configuration is an equilibrium configuration. In the unstable equilibrium configuration, there exist arbitrarily small disturbances under which the system moves to a new (not necessary stable) equilibrium configuration.

The Lagrange-Dirichlet theorem states that a minimum of the potential energy in a conservative mechanical system is a stable equilibrium configuration. As the potential energy for linear analysis is given by $W=$ $\frac{1}{2}\left(\mathbf{Y}^{T} \cdot \mathbf{K}_{0} \cdot \mathbf{Y}\right)$, a correspondence can be found between the positiveness of the tangent stiffness matrix

$$
\mathbf{K}_{T}(\mathbf{Y})=\frac{\partial \mathbf{K}}{\partial \mathbf{Y}}
$$

and the system stability. This is then represented by the sign of the eigenvalues, as summarized in Table 4.

To illustrate this discussion, the sail-type geometry shown in Figure 13 has been loaded with a typical constant pressure $\left(10^{-4} \mathrm{~N} / \mathrm{mm}^{2}\right)$ and analysed in order to detect the equilibrium behaviour in relation to the generation of wrinkles. A mesh with about 8000 elements was chosen, with 86 elements per horizontal section and some adequate refinement near the corners. This was judged a sufficient mesh resolution for a limited number of eigenvalues. 
Table 4: Equilibrium and eigenvalues of the tangent stiffness matrix

\begin{tabular}{|c|c|c|}
\hline Matrix property & Eigenvalues & Equilibrium \\
\hline \hline Positive definite & $\lambda_{i}>0 \forall i=1, n d o f$ & Stable \\
\hline Non-positive definite & $\lambda_{j}<0$ for some $j$ & Unstable \\
\hline Semi-positive & $\lambda_{i} \geqslant 0 \forall i$ and $\lambda_{j}=0$ for some $j$ & Critical \\
\hline
\end{tabular}

Table 5: Parameters used in the eigenvalue analysis

\begin{tabular}{|c|c|c|c|c|c|c|}
\hline Radius & Width angle & N. elts. & $t$ & $E$ & $\nu$ & $\rho_{s}$ \\
\hline$[\mathrm{mm}]$ & {$[\mathrm{Rad}]$} & {$[-]$} & {$[\mathrm{mm}]$} & {$\left[\mathrm{N} / \mathrm{mm}^{2}\right]$} & {$[-]$} & {$\left[\mathrm{Kg} / \mathrm{m}^{3}\right]$} \\
\hline 1000 & $\pi / 5$ & 8000 & 0.05 & 376 & 0.4 & 1.15 \\
\hline
\end{tabular}

Of course, after discretization the number of eigenmodes is finite unlike for the continuous case, and is equal to the number of degrees of freedom Ndof of the structure. The analysis specifications are summarized in Table 5. The physical quantities selected - namely the thickness $t$, Young's modulus $E$, the Poisson ratio $\nu$ and the mass per unit volume $\rho_{s}$ - are typical of sail-type materials. Except when otherwise clearly specified, all the numerical values used in the paper refer to those given here. Note that we will always neglect the effect of the sail self-weight, since it is roughly two orders of magnitude below that of the pressure loading.

The tangent stiffness matrix was extracted every 20 time steps, and an eigen-analysis was performed for the first 10 eigenmodes of lowest associated frequencies. Figure 14-top plots the horizontal displacement of a sample node located on the structure symmetry line at height $z=750 \mathrm{~mm}$, while Figure 14-bottom shows the evolution of the first eigenvalue. Instability is soon encountered after starting the calculation process, and it only disappears near the end of the time path, when the structure is established in a stable configuration. This instability is the cause of the wrinkling generation, as it appears by inspecting the eigenvectors during the time path. Figure 15 displays the first eigenvector for time steps 120, 236 and 394, all representing unstable modes. In the first case, the unstable mode spreads to the whole sail. As the calculation proceeds, the instability tends to concentrate in the wrinkled zones. At the end of the calculation no unstable mode remains, but the first eigenmodes still represent wrinkling patterns. This indicates 


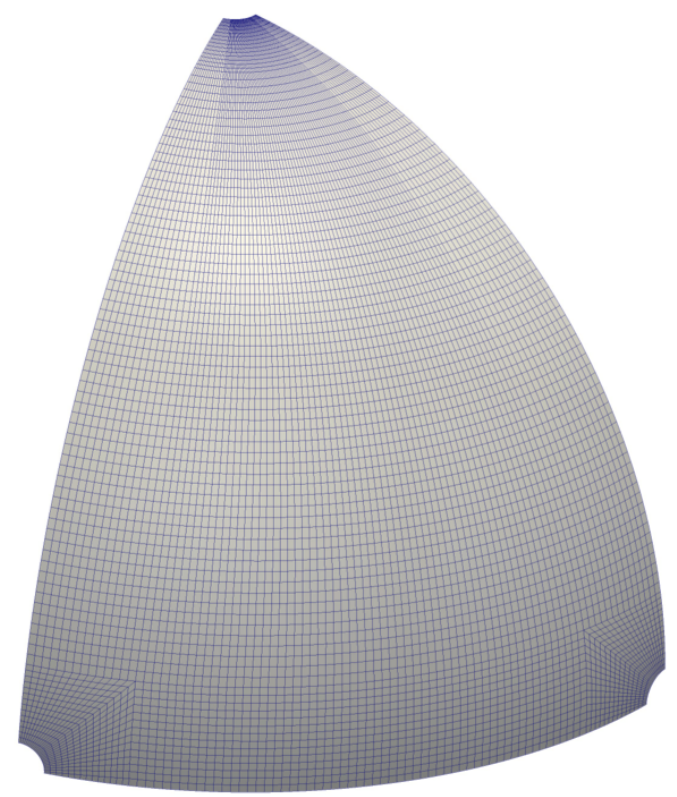

Figure 13: Gennaker-like geometry considered in the stability analysis, see Table 5 for detailed specifications

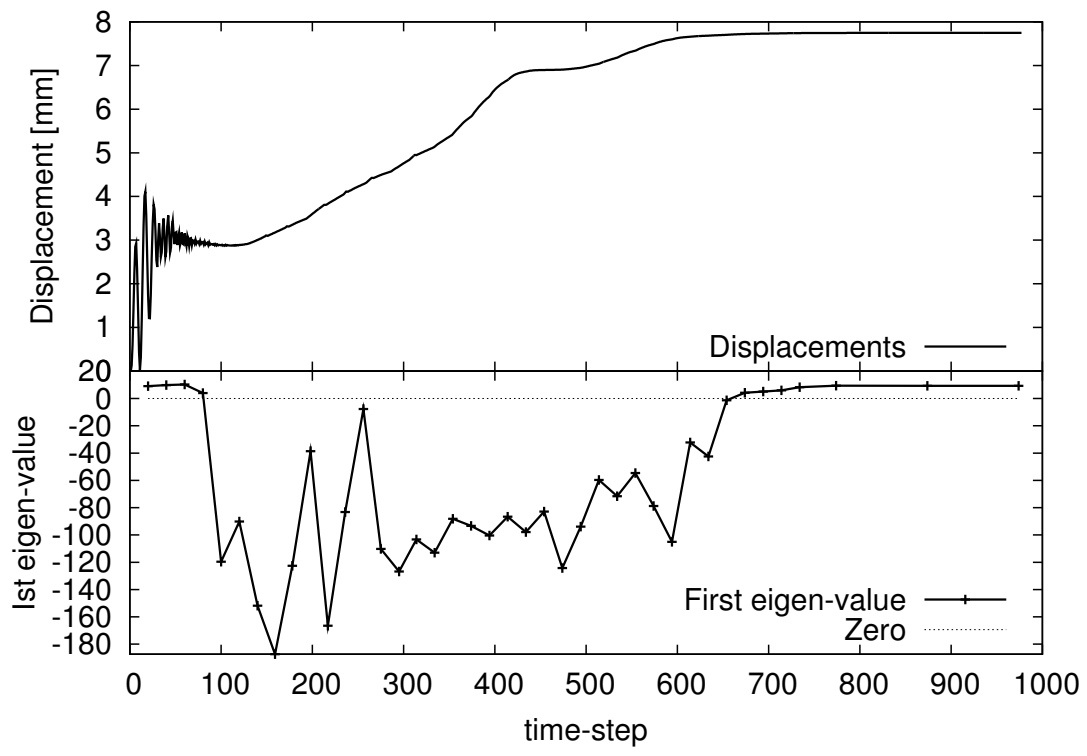

Figure 14: Top: Sample node horizontal displacement path; Bottom: First eigenvalue along deformation path 


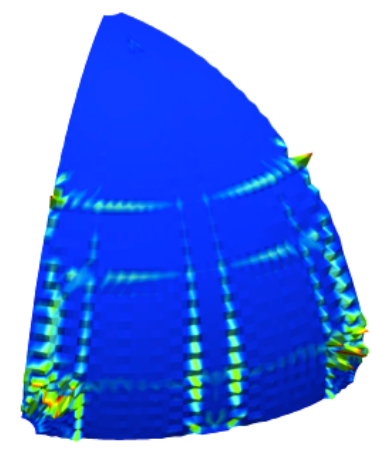

Time step 120

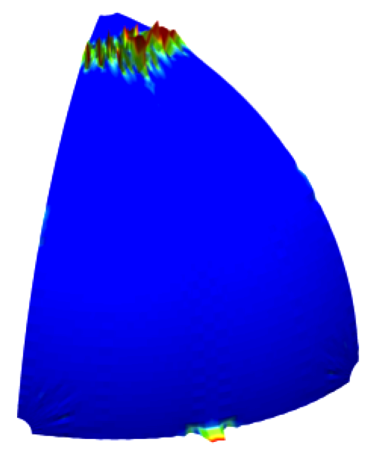

Time step 236

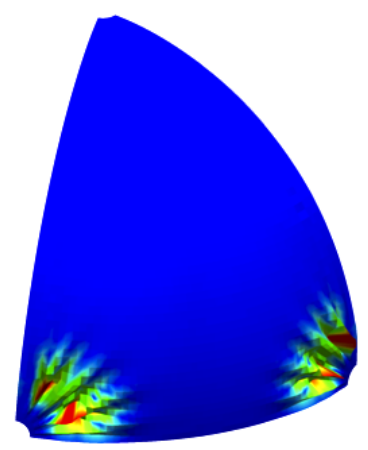

Time step 394

Figure 15: First eigenvector plot for three time-steps

the dynamics of the wrinkling generation, as it appears to play the role of absorbing the instabilities in well-circumscribed zones.

The strong link between instability and wrinkling is confirmed when inspecting a number of eigenmodes for a single time step. Taking for instance the $394^{\text {th }}$ time step, in the middle of the unstable time period, the majority of the first eigenvectors represent the wrinkling deformation components, as shown in Figure 16 where the first six eigenvectors are reported.

\section{Mesh sensitivity in the wrinkling representation}

A parametric grid sensitivity study was undertaken in order to assess the adequate grid fineness for accurately capturing the wrinkles. The purpose was also to verify whether the converging behaviour of the finite element solution is affected by the wrinkling development.

Two geometries and 14 meshes per geometry have been adopted for this mesh sensitivity analysis. In the first geometry, a Gennaker-like structure is analysed, and the geometry is described using a spherical sector of radius $R=$ $1000[\mathrm{~mm}]$, and of height and width corresponding to angles $\pi / 2$ and $2 \pi / 5$, respectively. For symmetry reasons, we will consider in our computations only half of this geometry along the width direction, and we display this reduced geometry in Figure 18. In fact, unless otherwise clearly specified all the geometries visualized in the rest of the paper represent half of the 


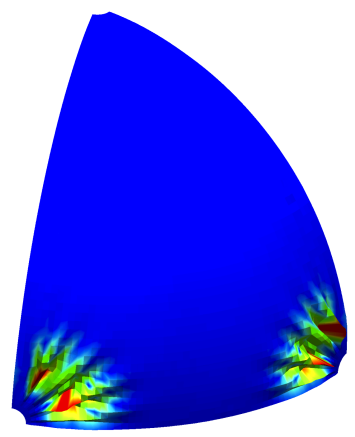

Time step 394 - Mode 1

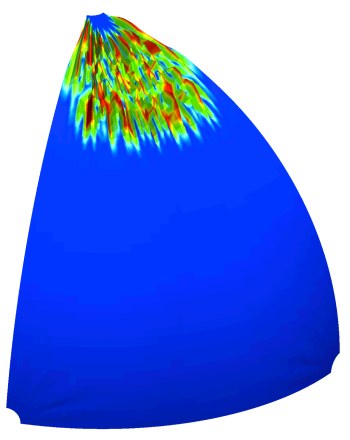

Time step 394 - Mode 4

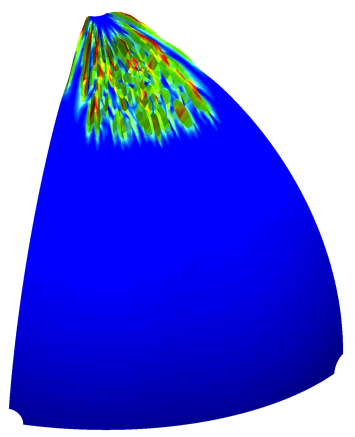

Time step 394 - Mode 2

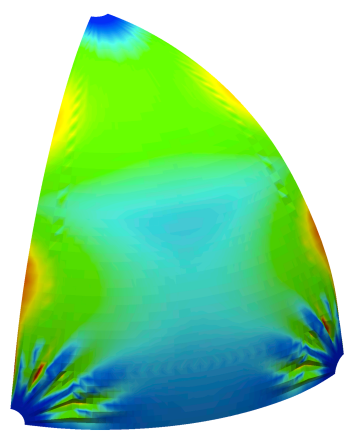

Time step 394 - Mode 5

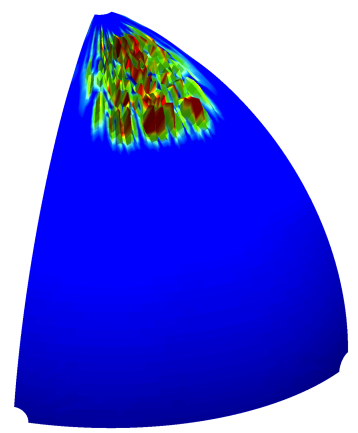

Time step 394 - Mode 3

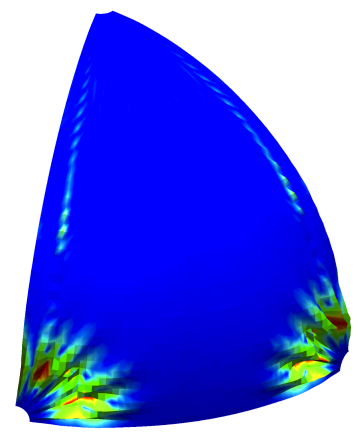

Time step 394 - Mode 6

Figure 16: First six eigenvectors at time-step 394 
real geometry. Of course, in actual applications, sail shapes will very seldom enjoy such symmetry properties, and at the very least suffer from geometric imperfections, indeed. Nevertheless, we consider here these generic shapes as part of a proof-of-concept study. The second shape analysed has the same spherical part with a cylinder added underneath, in order to produce a Spinnaker-like structure as displayed in Figure 22. The height of this cylinder is equal to the radius of the spherical component. In all the meshes the element size distribution was kept as homogeneous as possible. However, the meshes were designed with finer discretization in regions close to the corners, where wrinkling generation is expected. The characteristics of these meshes are reported in Tables 6 and 7 .

Table 6: Meshes specifications for the Gennaker mesh sensitivity analysis. Ne is the number of elements, $N p$ the number of nodes and $E p S$ the number of element per horizontal section

\begin{tabular}{|c|c|c|c|c|c|c|c|c|}
\hline Mesh & $m_{2}$ & $m_{3}$ & $m_{5}$ & $m_{10}$ & $m_{15}$ & $m_{20}$ & $m_{25}$ & $m_{30}$ \\
\hline$N e$ & 49 & 130 & 406 & 1761 & 4066 & 7321 & 11526 & 16681 \\
\hline$N p$ & 67 & 158 & 405 & 1859 & 4214 & 7519 & 11774 & 16979 \\
\hline$E p S$ & 4 & 7 & 13 & 28 & 43 & 58 & 73 & 88 \\
\hline \hline Mesh & $m_{35}$ & $m_{40}$ & $m_{45}$ & $m_{50}$ & $m_{55}$ & $m_{60}$ & & \\
\hline$N e$ & 22786 & 29841 & 37846 & 46801 & 56706 & 67561 & & \\
\hline$N p$ & 23134 & 30239 & 38294 & 47299 & 57254 & 68159 & & \\
\hline$E p S$ & 103 & 118 & 133 & 148 & 163 & 178 & & \\
\hline
\end{tabular}

For the Gennaker type geometry, we selected two specific cross-sections for their interest regarding the wrinkling pattern, see Figure 17. The Head section is characterized by the intersection of the deformed shape with a plane, the normal vector of which is tangent to the surface and directed along the bisector line, while the height of the cross-section is defined such that approximately half of the wrinkle length is intersected. The Corner crosssection is defined by intersecting the structure with a plane passing through a point $Q$ located on the bottom edge at the crossing with the bisector line. The plane is normal to a vector, the horizontal projection of which is tangent to the sail at the point $Q$, but vertically elevated at $45^{\circ}$ upwards.

Figure $19 a$ shows the results in terms of displacements of the selected points shown in Figure 17, and Figure $19 b$ shows the number of wrinkles for the Gennaker geometry. Acceptable results for the prediction of local wrin- 


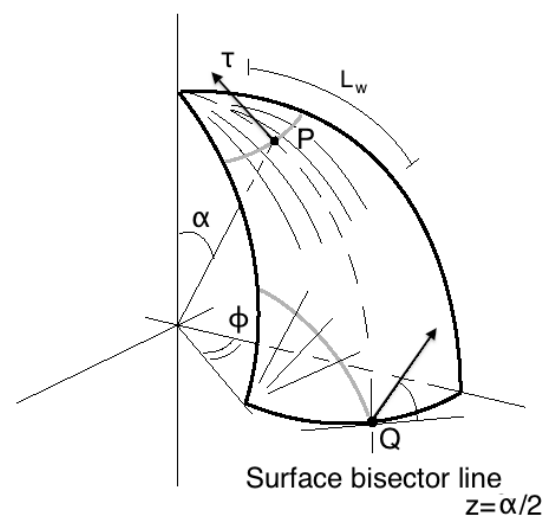

Figure 17: Definitions used for identifying the Head and Corner sections

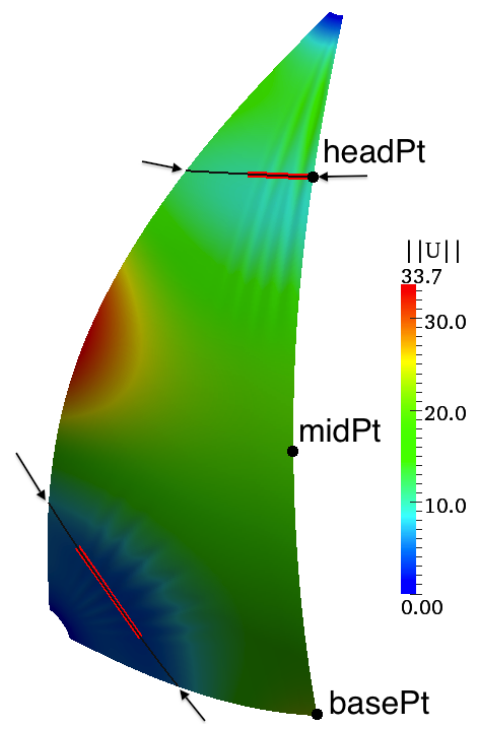

Figure 18: Gennaker geometry with Head and Corner cross-sections, with parts represented in Figures $20 b$ and $21 b$ highlighted in red

kling are obtained starting from the mesh $m_{45}$, which has about 20 elements per wrinkle in the Head section. This result is in line with that obtained in Section 3. Regarding the number of wrinkles captured with different meshes, it is interesting to observe the general behaviour of the curves, where discontinuities are observed when the mesh considered is able to capture new wrinkles of smaller dimensions. This is better seen in Figures 20-21, where 

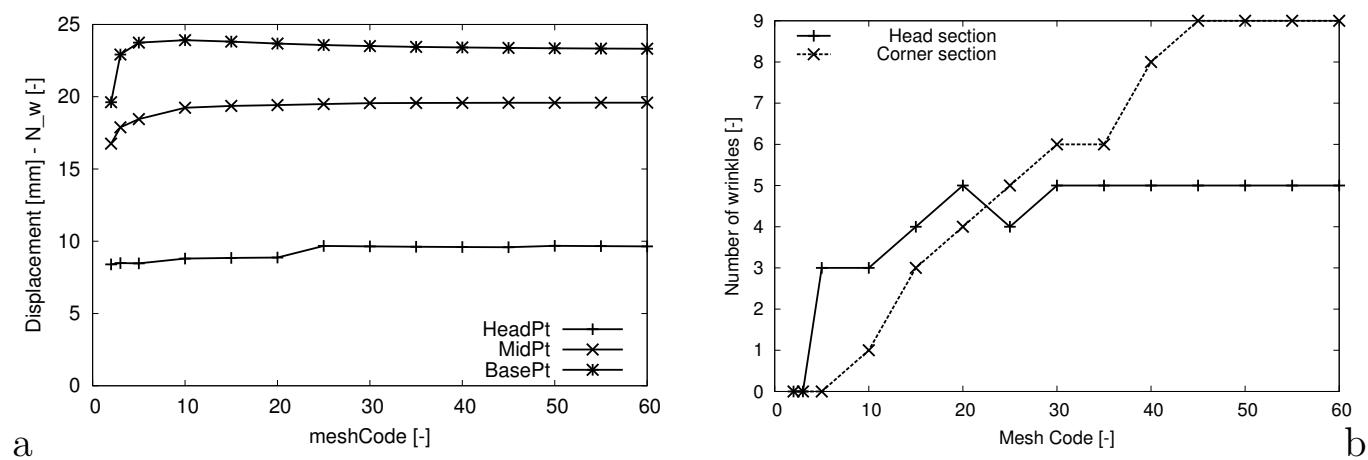

Figure 19: Gennaker shape - a: Mesh impact on point-wise displacements. $b$ : Mesh sensitivity in the number of wrinkles
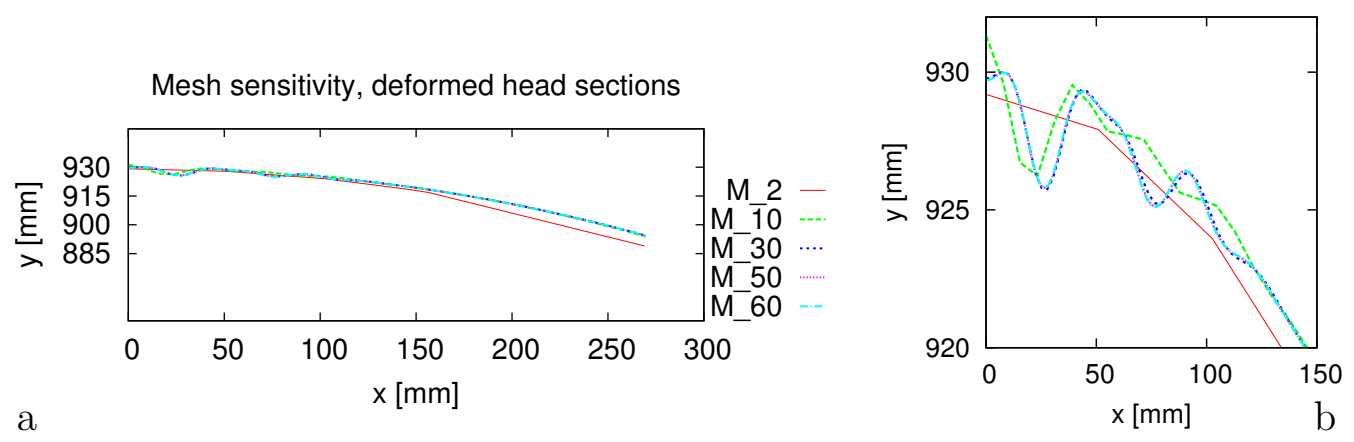

Figure 20: Gennaker shape mesh sensitivity - Head sections. $a$ : Global view and $b$ : Zoom on the wrinkled region
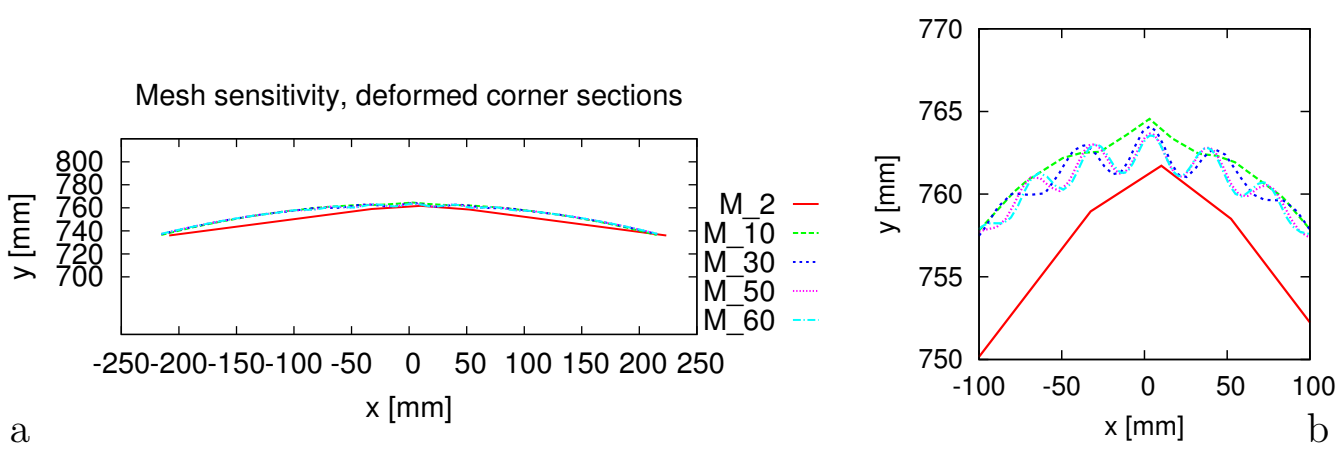

Figure 21: Gennaker shape mesh sensitivity - Corner sections. $a$ : Global view and $b$ : Zoom on the wrinkled region 
deformed cross-sections are displayed for meshes of increasing accuracy. Note in particular in Figure $21 b$ how two small additional wrinkles are captured in the finest meshes, whereas the overall behaviour is well represented for coarser meshes.

Table 7: Mesh specification for the Spinnaker mesh sensitivity analysis. $N e$ is the number of elements, $N p$ the number of nodes and $E p S$ the number of element per horizontal section

\begin{tabular}{|c|c|c|c|c|c|c|c|}
\hline Mesh & $m_{3}$ & $m_{5}$ & $m_{10}$ & $m_{15}$ & $m_{20}$ & $m_{25}$ & $m_{30}$ \\
\hline$N e$ & 24 & 104 & 513 & 1024 & 22203 & 3552 & 5133 \\
\hline$N p$ & 36 & 128 & 565 & 1283 & 2330 & 3687 & 5250 \\
\hline$E p S$ & 4 & 8 & 18 & 28 & 38 & 48 & 58 \\
\hline \hline Mesh & $m_{35}$ & $m_{40}$ & $m_{45}$ & $m_{50}$ & $m_{55}$ & $m_{60}$ & \\
\hline$N e$ & 7072 & 9477 & 12848 & 16023 & 20088 & 23423 & \\
\hline$N p$ & 7262 & 9634 & 13105 & 16310 & 20410 & 23770 & \\
\hline$E p S$ & 68 & 78 & 88 & 98 & 108 & 118 & \\
\hline
\end{tabular}

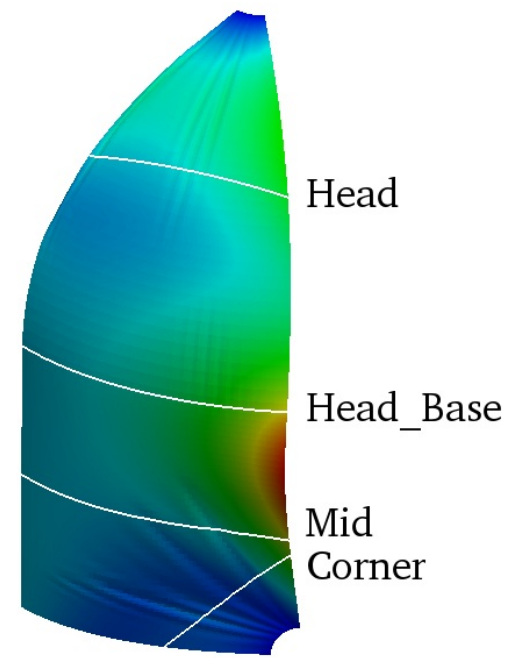

Figure 22: Spinnaker geometry with the trace of the sections used for the evaluations

Similar conclusions can be drawn in the case of the Spinnaker geometry, see Figures 23-25 with the cross-sections defined in Figure 22. In this case the overall displacements obtained are larger, since the sail is constrained at 

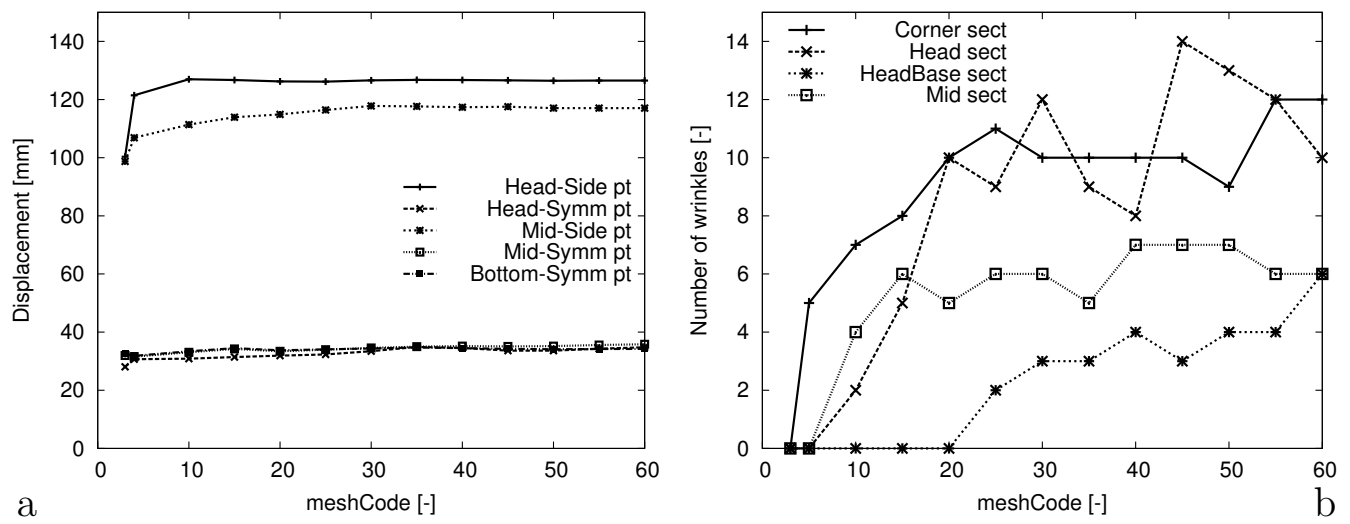

Figure 23: Spinnaker shape $-a$ : Mesh impact on point-wise displacements. $b$ : Mesh sensitivity in the number of wrinkles
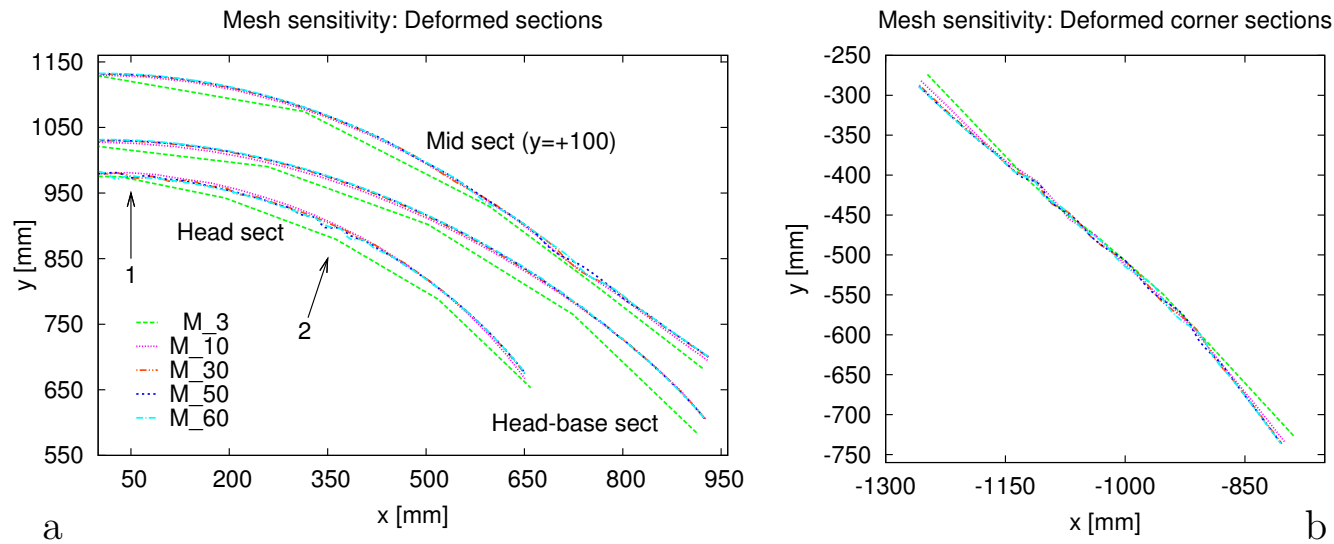

Figure 24: Spinnaker shape mesh sensitivity - a: Head, Head-base and Mid sections; $b$ : Corner sections

points farther away from each other, and developable surfaces - referring to the cylindrical part, here - tend to undergo larger motions. Regarding the wrinkling representation, we note an adequate convergence with the sequence of meshes, except specifically in the Head cross-section. Although the curves in Figure 23 do not look well converged, some limited oscillations of the wrinkling count are due to the definition of the wrinkles recognition algorithm criterion, since in some cases the thresholds are reached or missed by a very small margin, while clear trends can be identified regarding the - hence approximate - number of wrinkles. In fact, this is the case for all sections 

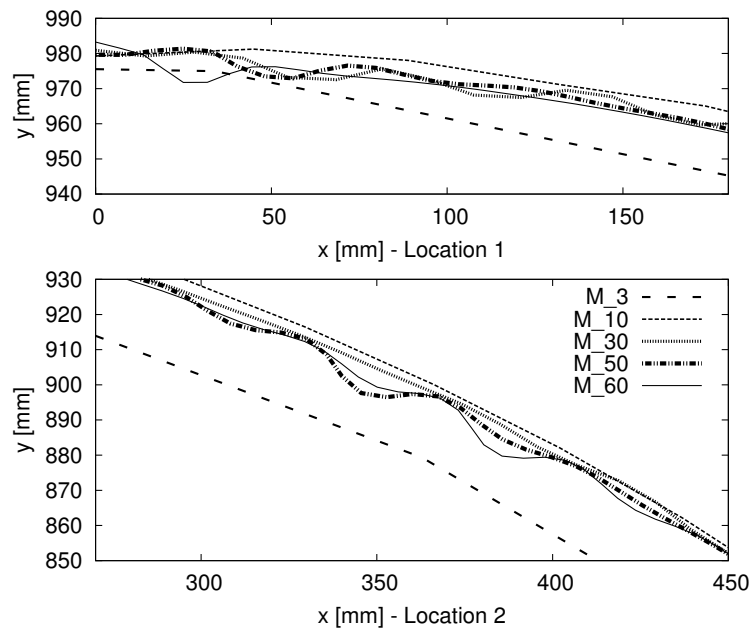

Figure 25: Spinnaker shape mesh sensitivity - Zoom on the wrinkles of the Head section, as specified by the arrows in Figure 24. Top: Region near the symmetry centerline. Bottom: Region near the half-side of the structure

except the Head section, which displays a high variability in the number of wrinkles. This is due to the progressive resolution of small amplitude wrinkles as the mesh becomes finer, see in particular Figure 25. We further discuss this issue in Section 8.

For both the Gennaker and the Spinnaker cases, the mesh sensitivity analysis indicate that the wrinkling is a higher-order phenomenon, hence a relatively coarse mesh is able to represent the general behaviour of the sail, whereas a detailed wrinkling representation requires at least 20 elements per wrinkle.

\section{Influence of material properties variations}

A set of tests was carried out using the Gennaker geometry and nine different combinations of material properties, all of which are realistic for sailtype materials. The parametric changes concerned Young's modulus and the thickness. Starting with an initial value, both Young's modulus and thickness parameters were multiplied and divided by a factor two, thus defining the test grid reported in Table 8. Based on the results of the mesh sensitivity analysis, it was then decided to use the mesh referenced as 45 for the 
following evaluations, since this mesh provides good convergence properties of the solution, recall Figure 19.

Table 8: Material test matrix (Analysis code number)

\begin{tabular}{|c|c|c|c|}
\hline$E\left[\mathrm{~N} / \mathrm{mm}^{2}\right] \quad t[\mathrm{~mm}]$ & 0.05 & 0.1 & 0.2 \\
\hline 188 & 1 & 2 & 3 \\
\hline 376 & 4 & 5 & 6 \\
\hline 752 & 7 & 8 & 9 \\
\hline
\end{tabular}
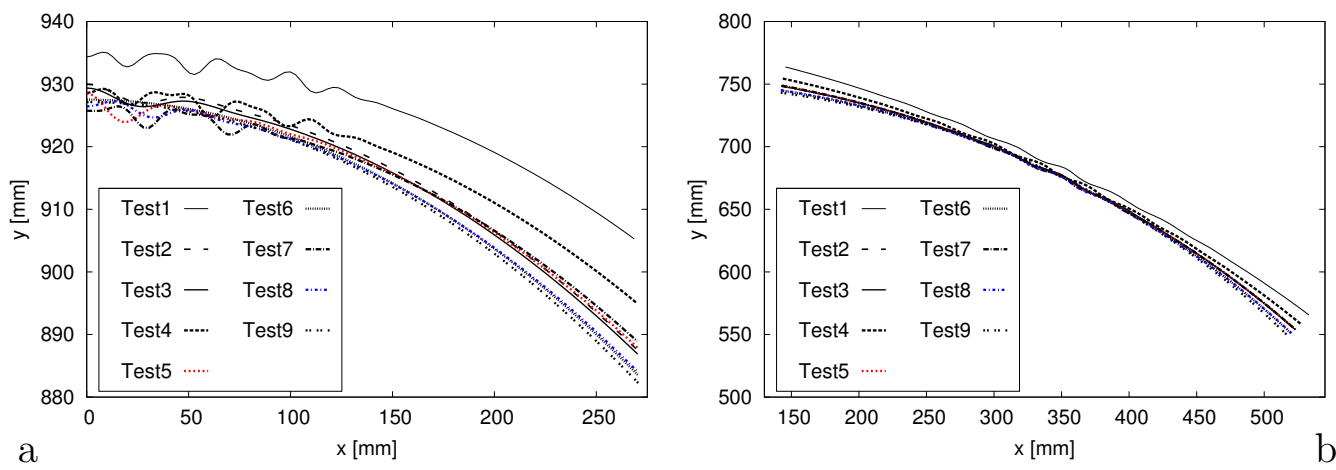

Figure 26: Parametric analysis for Gennaker shape - a: Head and b: Corner deformed sections for test cases referenced in Table 8
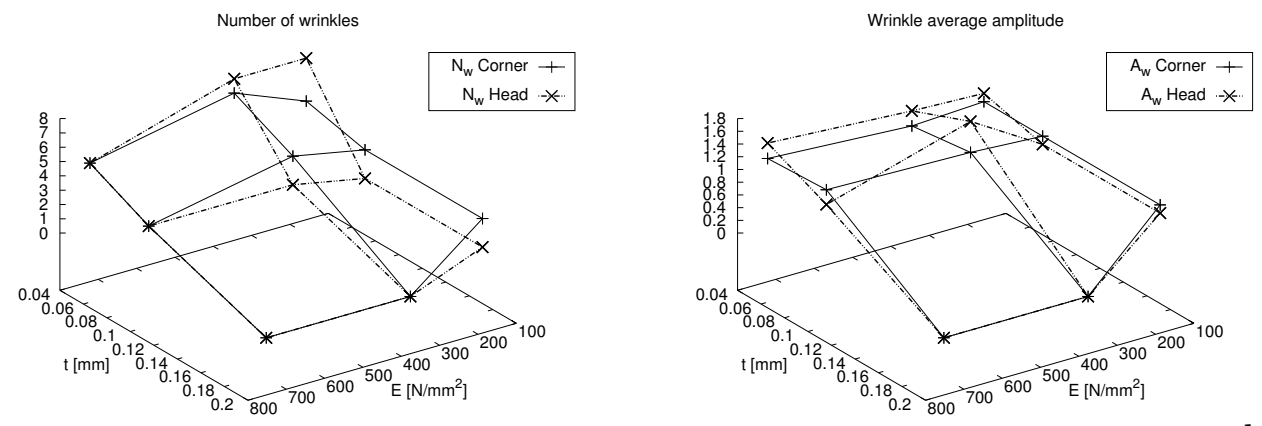

a

Figure 27: Parametric analysis for Gennaker shape - $a$ : Number of wrinkles and $b$ : Average amplitude of the wrinkles 

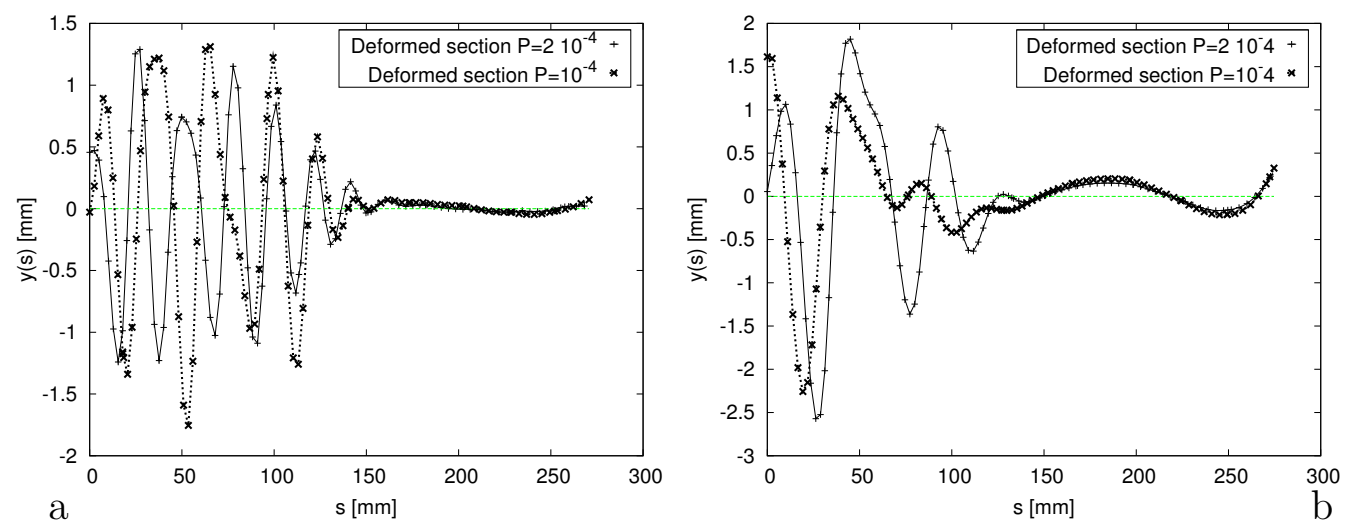

Figure 28: Influence of the load for Gennaker shape: homologous Head sections when doubling the load -a: Case $_{1}$ and $b:$ Case $_{5}$

The same two probe Head and Corner cross-sections were considered as in Section 5. The deformed cross-sections are visualized in Figure 26, and the corresponding wrinkling indicators are plotted in Figure 27. Although no specific relationship can be identified regarding the dependency of these indicators with respect to the parameters considered, this study shows that our procedure can adequately capture the wrinkling behaviour for the whole parametric range of concern. Globally, the amplitude and the number of wrinkles are principally influenced by the thickness parameter, and as expected the thinner the material the larger the number and the amplitude of the wrinkles, for a given pressure load. By contrast, no clear general trend can be observed when varying Young's modulus.

A comparison can then be made when doubling the load. In this case the wrinkling pattern is not strongly affected, and similar features can be identified. This comparison is shown in Figure 28 for cases number 1 and 5, representative of the whole spectrum of analysed cases. We observe that the deformed sections preserve the same essential features, thus with a stronger dependency upon the material properties than on the loading magnitude.

\section{Influence of the geometry}

In this Section we investigate the influence of the geometry on the wrinkling development, starting with Gennaker-like shapes of varying widths, and proceeding with Spinnaker-like shapes of various heights. 


\subsection{Spherical shape with varying width}

Table 9: Specifications of the analysed geometries

\begin{tabular}{|c|c|c|c|c|c|}
\hline$\phi$ & $\pi / 2$ & $\pi / 3$ & $\pi / 4$ & $\pi / 5$ & $\pi / 6$ \\
\hline$E p S$ & 225 & 150 & 111 & 90 & 75 \\
\hline
\end{tabular}
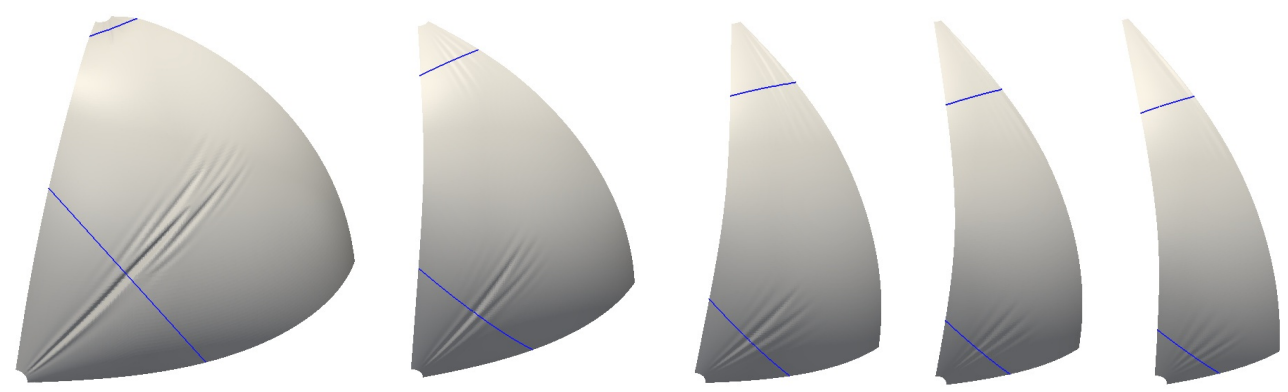

Figure 29: Five different analysed geometries (symmetry) with the trace of the sections used for the analysis

Five different spherical geometries were analysed for this investigation of the effect of the shape on wrinkling development. These geometries were derived from that already analysed in Section 5 by varying the half-width angle $\phi$ previously defined as $\pi / 5$, see Figure 29 . The mesh density was kept as constant as possible by preserving the ratio of number of element by width angle, which results in the number of elements per horizontal section $E p S$ listed in Table 9. All physical quantities remained set as in Case 5 of Table 8.

The resulting computed deformed Head cross-sections - as identified in Figure 17 - are shown in Figure 30. We first observe that all cross-sections have roughly similar extents, which is informative as regards the location of the wrinkles. More generally, the shapes of the deformed Head and Corner cross-sections - wrinkling included - are quite comparable for the various geometries, except for $\phi=\pi / 2$ in the Head section for which the global curvature is inverted, indeed. This behaviour can be explained by observing that the extension of the wrinkles is in this case very small, hence the crosssection is located very near the attachment and the force transmission area associated with the wrinkles tends to pull the sail downwards.

In the Corner section, the number of wrinkles as well as the average wrinkle amplitude are largely influenced by the base angle. As expected, the 

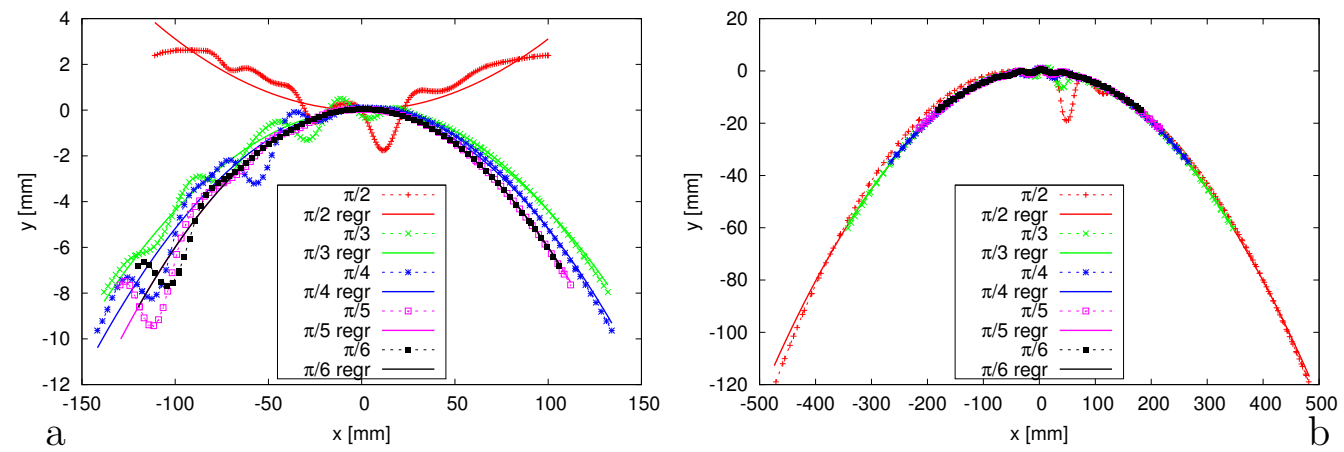

Figure 30: Gennaker shape with varying base angle $-a$ : Head and $b$ : Corner deformed sections with corresponding regressions

wider the angle - i.e. the larger the structure - the higher the number and amplitude of wrinkles, see also Figure 29. Concerning the Head sections, the wrinkles present a more stable behaviour, both in terms of number and of amplitude.

The general wrinkling behaviour, which can be observed in the screen shot in Figure 29, is well represented by the wrinkling indicators in Figure 31. In particular the wrinkling index indicates well the large increase in the wrinkling development on the Corner section as the geometry becomes wider.

\subsection{Spinnaker-like shapes with varying heights}

Here we consider Spinnaker-like shapes similar to that defined in Section 5 and vary the height $h$ of the cylindrical part with values $h / R=1 ; 1.5 ; 2$, in addition to the previous case $h / R=0.5$. All other quantities remain unchanged. Results are reported in terms of the deformed sections already shown in Figure 22, where the Head-base section is always defined as the junction of the spherical and cylindrical parts, and the so-called Mid section is taken at half the height of the cylindrical part.

As we increase the height of the cylindrical body, the overall pattern formed by the displacements - namely, along the force application direction in the lower part of the structure, and in the opposite direction in the higher part - is more and more marked, see Figures 32 and 33. In addition, in the higher part the spherical shape progressively becomes flatter as the width decreases due to the distance to the lower boundary conditions, while sharp wrinkles appear and contribute to the overall width reduction, indeed. The wrinkling behaviour is analysed in more detail in Figure 34 which provides 

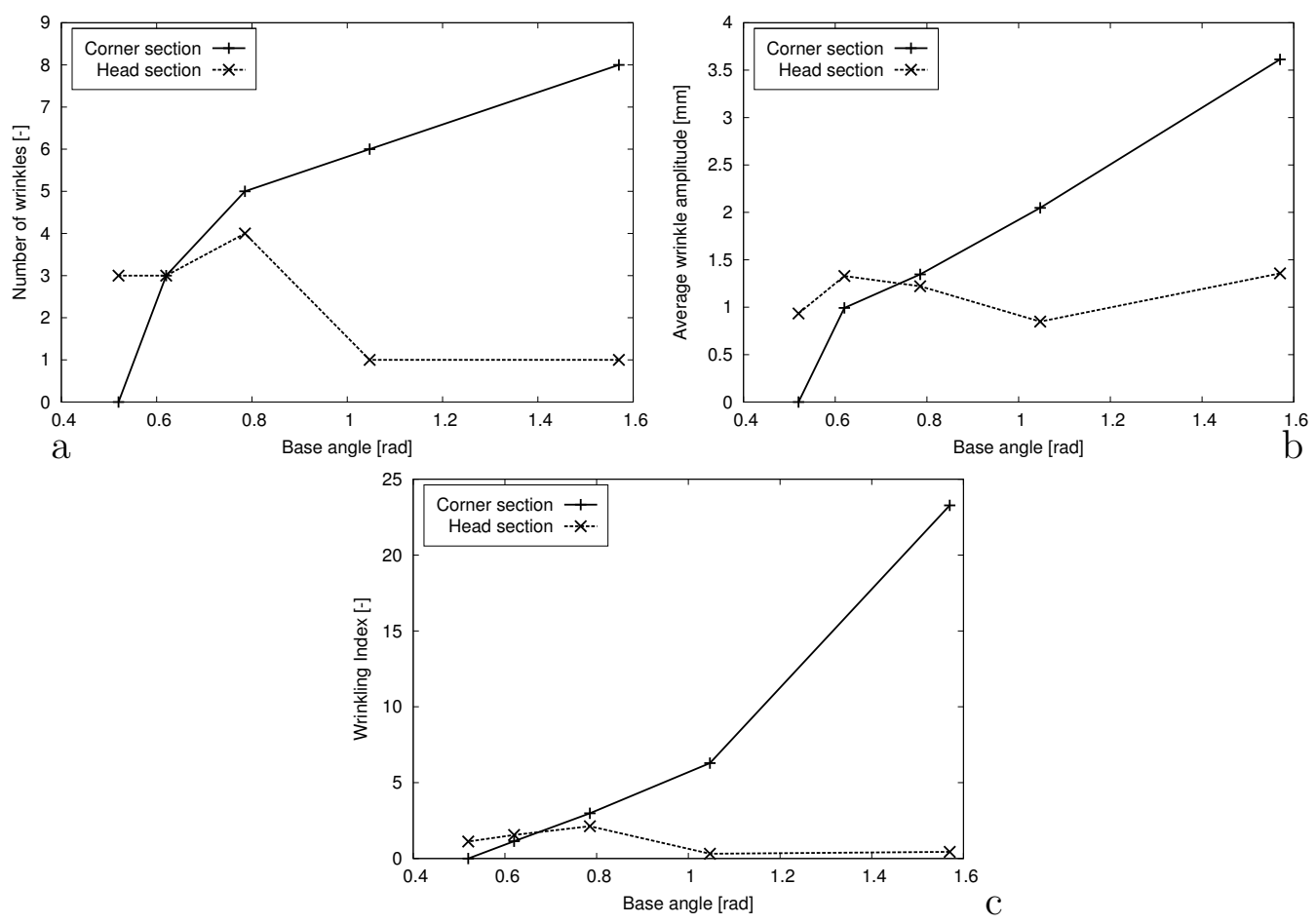

Figure 31: Gennaker shape with varying base angle - $a$ : Number, $b$ : Average amplitude and $c$ : Wrinkling index of wrinkles for the Head and the Corner sections

for each shape and section the number, the average amplitude of wrinkles, recall Figure 3, and the Wrinkling index value.

Analysing these indicators in conjunction with the deformed shapes visualized in Figure 32, we see that the mean amplitude of the wrinkles increases with the height of the cylindrical part. Only the Head-Base section constitutes an exception, which occurs because the wrinkling is so developed in this junction region that some wrinkles tend to roll-over, thus inducing very small amplitude oscillations. This reveals an area of very low physical stability, in which the wrinkling is insufficiently captured by the mesh. The response in terms of the number of wrinkles - see Figure $34 a$ - tends to be more stable. Here also, the number of wrinkles for the Head-Base section constitutes an exception, as it rapidly increases then stabilizes around much higher values than in the other sections.

The general behaviour of the curves for the Wrinkling Index - see Figure $34 c-$ follows the number of wrinkles. The response is in this case slightly 

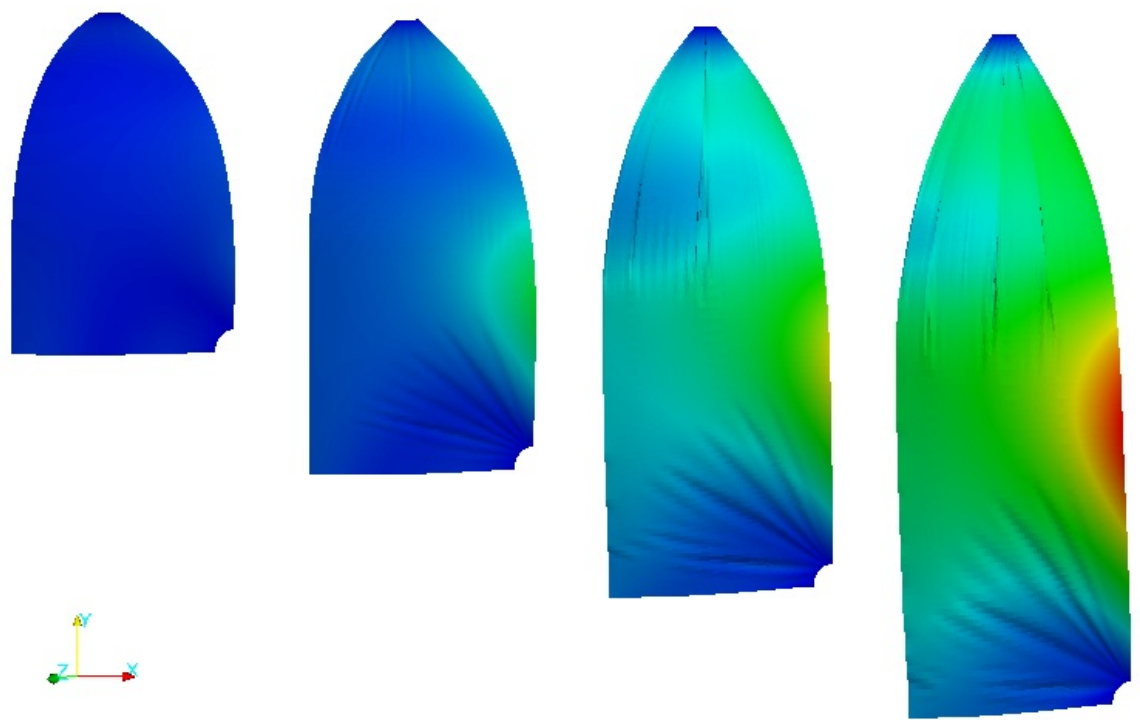

Figure 32: Spinnaker-like deformed shapes with varying height parameter $h / R=$ $0.5 ; 1 ; 1.5 ; 2$ (left to right)
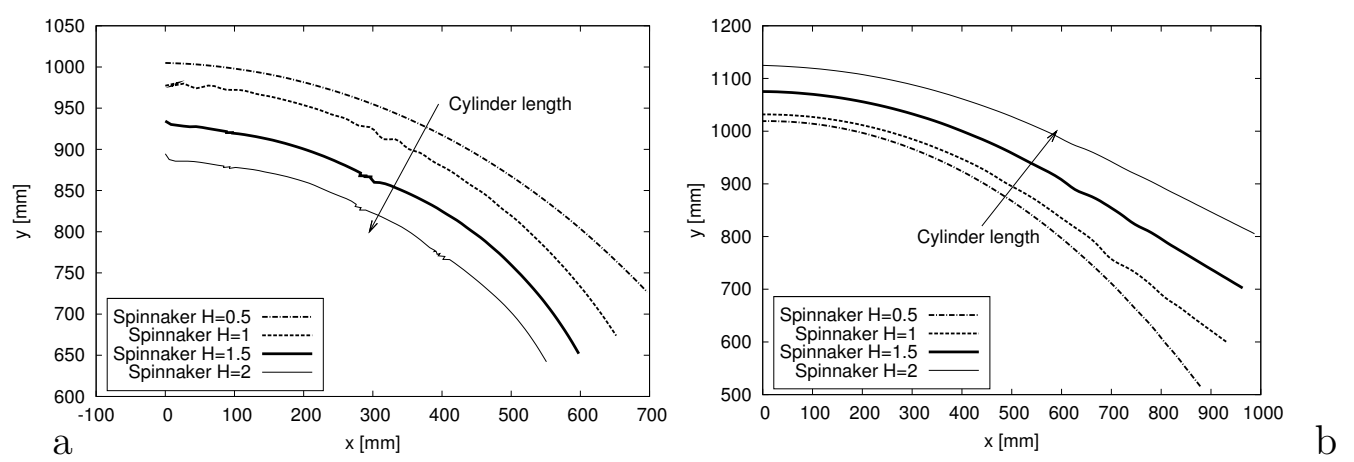

Figure 33: Spinnaker shape with varying cylinder length $-a$ : Head and $b$ : Head-base deformed sections

more regular, and it respects the general wrinkling behaviour observed in Figure 32.

\section{Discussion}

First of all, it should be emphasized that the general deformation pattern of a sail is well represented, in particular with the wrinkling developing for 

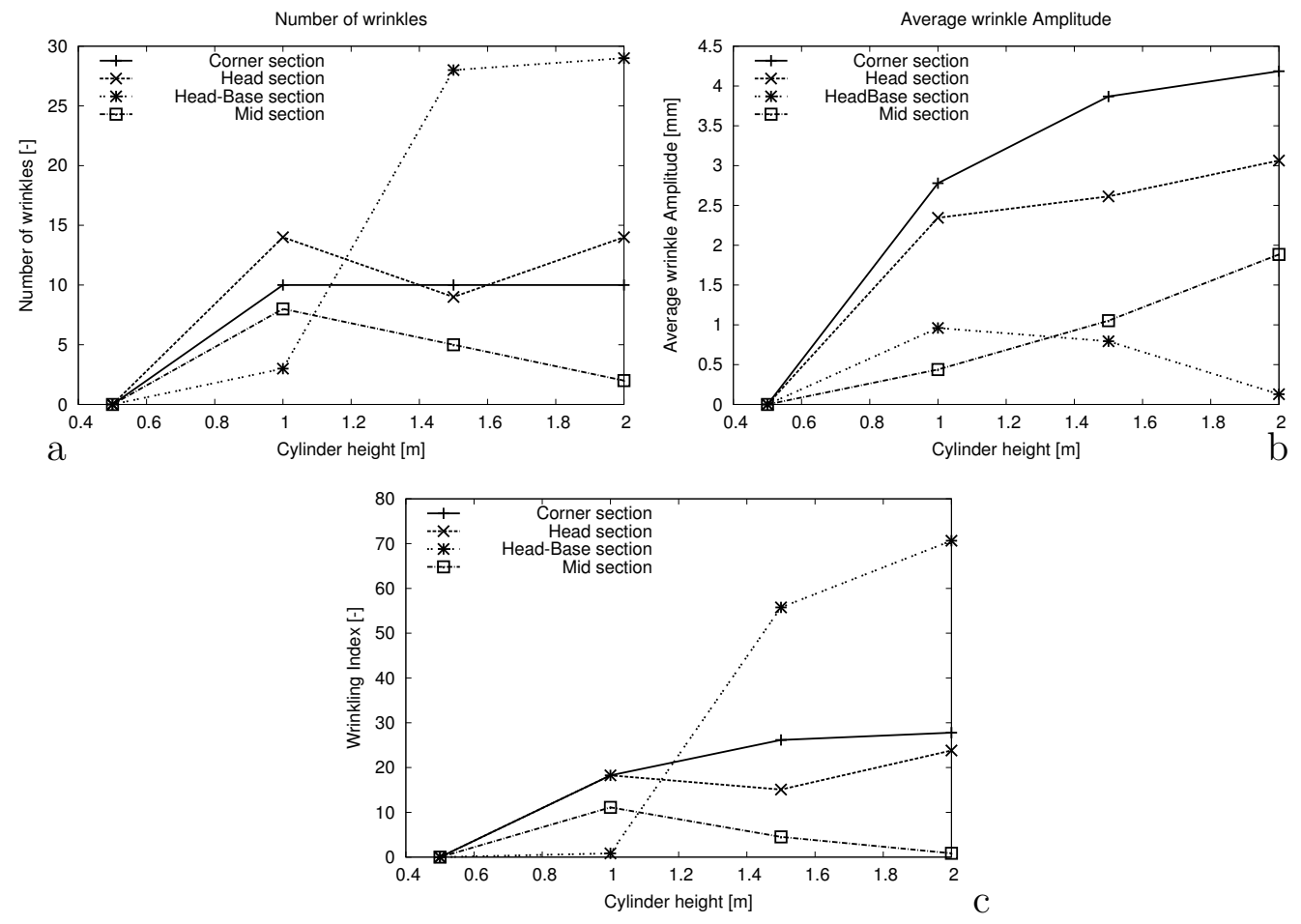

Figure 34: Spinnaker shape with varying cylinder length $-a$ : Number, $b$ : Average amplitude and $c$ : Wrinkling Index of the identified wrinkles

about a third of the structure on both the head and the constrained corner. Geometrically, sails made of a spherical head and a cylindrical base are those where the wrinkles determine a large change in the global flying shape. In such configurations large wrinkles arise at the junction between the spherical and the cylindrical parts, and this induces a shrinkage which significantly changes the extension of the structure head. Moreover, we have observed a good convergence of the computed wrinkles when adequately refining the meshes, except in the case of the Spinnaker-like shapes with a slender cylindrical body. In particular, for the case considered in Section 5 namely, with $h / R=1$ - we have obtained a significant mesh dependence and a lack of resolution of the wrinkles including for the finest meshes considered, recall Table 7 and Figure 25.

In order to further analyse this case we display in Figure 35 the details of the deformed pattern by plotting the distance to the regression curve against the arc-length coordinate, for two of the finest meshes already considered. 


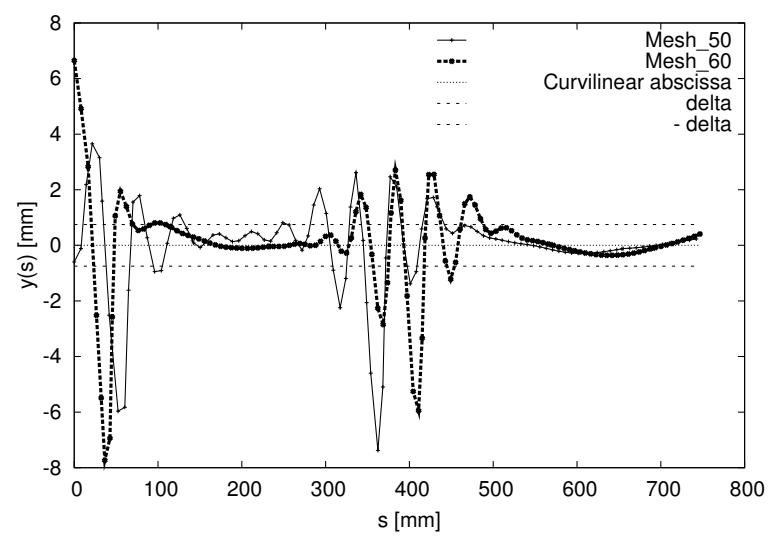

Figure 35: Spinnaker shape with $h / R=1$ : Head cross-section in arc-length representation for 2 fine meshes

We see that the numerical solutions consistently identify two main groups of wrinkles, albeit that within each group the individual wrinkles are insufficiently resolved, with just a few nodes per wrinkle even for the finest mesh. This indicates a rather low physical stability - locally - in this configuration due to the shape considered. Moreover, as the wrinkling wavelength is consistently estimated with the three meshes, we can conjecture that increasing the mesh density by a factor $3-5$ across the wrinkles would produce an accurate prediction of the phenomenon. Of course, refining the mesh uniformly and in all directions by such a factor would lead to inordinately expensive computations, hence the mesh adaptation should be performed locally and in a non-isotropic manner, which is out of the scope of our analysis.

This phenomenon is even more pronounced for Spinnaker shapes with a higher cylindrical body, as exemplified in Figure 36. In this case, some rather deep and rapidly oscillating wrinkles appear around the middle of the section, and here clearly much more refined meshes would be required to resolve these features, probably also with self-contact analysis - and possibly bifurcation analysis - involved.

\section{Concluding remarks}

A dynamic Finite Element method has been used for the analysis of downwind sail-type structures. The main purpose was here to investigate the development of wrinkling, a phenomenon commonly observed in practice 


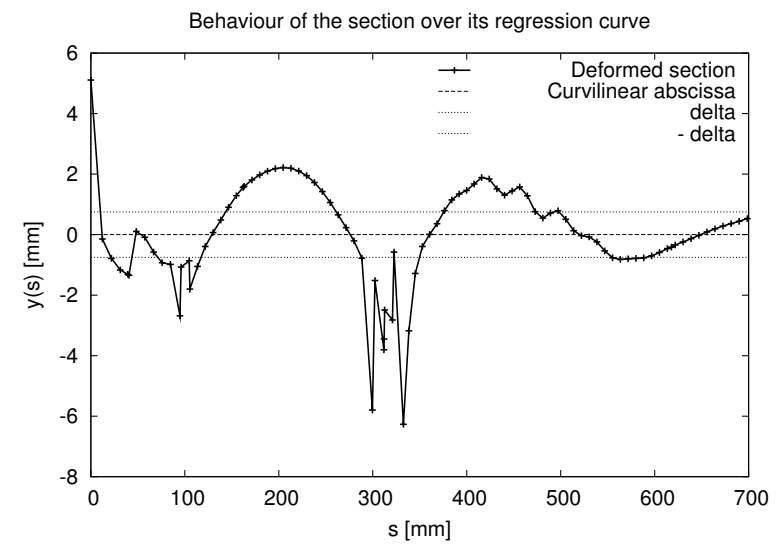

Figure 36: Spinnaker shape with $h / R=1.5$ : Head cross-section in arc-length representation for computational mesh

for such structures. Considering the wrinkling in this type of analysis is of great interest, since wrinkling affects the stress distribution in the fabric.

The analysis was carried out using non-linear MITC shell finite elements. The main advantage of using a shell model - instead of a membrane model - is that no ad hoc wrinkling model is required, as wrinkles naturally appear on the deformed surface and are controlled by a balance of membrane and bending stresses. The solution was obtained using a dynamic scheme with Rayleigh damping, able to provide sufficient stability in the numerical analysis. Instability can in fact arise due to the effect of the fabric thickness, which is usually very small compared to the other dimensions of the structure.

A validation test case was reproduced and excellent agreement was found between the numerical and the experimental results. Compared to previous numerical solutions the present method has the advantage of not requiring any particular initialization.

Subsequently, realistic sail shapes were considered, with a prescribed pressure loading. In most cases we found that both the overall deformed configuration and the detailed wrinkling pattern could be adequately captured with meshes of reasonable sizes. For just a few geometries, however - corresponding to Spinnaker shapes with a long cylindrical body - we concluded from the detailed convergence analysis that, while the global flying shape was well reproduced, some very fine and highly oscillating wrinkling behaviour was clearly detected but would require a much denser mesh to resolve the phenomenon. For such computations, dynamically adaptive meshes and/or 
highly intensive computing would be needed, which was out of the scope of this paper.

Finally, we proposed an enhanced Wrinkling Index which allows to detect the general behaviour of a deformed section in terms of the wrinkling development. This index was shown to be a particularly valuable indicator for the mesh sensitivity and the parametric shape variation analysis.

Further developments primarily regard various refinements of the model, in order to represent some even more realistic sail configurations such as with non-isotropic material models, corner reinforced zones, and cable boundary conditions. Of course, another very important perspective concerns the use of such sail models coupled with the wind flow in a fluid-structure interaction framework. Whereas in this work a constant pressure loading has been used to represent the operating condition of the sail, in reality there is a complex interaction between the deformed shape - including wrinkles - and the distribution of the actual wind loading [26]. The strategy developed in this work provides a basis for coupled FSI analysis [25]. Of course, for an actual transient FSI analysis, the structural damping should be modeled and calibrated in a physical manner, and an adequate coupling strategy should be used to ensure the numerical stability of the coupled solution, see e.g. $[14,13]$.

\section{Bibliography}

[1] ABAQUS Theory and User's Manual, Version 6.2. Hibbitt, Karlsson, and Sorensen, Inc., Pawtucket, RI, USA, 2001.

[2] Vinicius Arcaro, Katalin Klinka, and Dario Gasparini. Finite element analysis for geometrical shape minimization. Journal of the International Association for Shell and Spatial Structures, 50(2), 2009.

[3] K.J. Bathe. Finite Element Procedures. Prentice Hall, 1996.

[4] K.J. Bathe, A. Iosilevich, and D. Chapelle. An evaluation of the MITC shell elements. Comput. \& Structures, 75(1):1-30, 2000.

[5] J.L. Batoz and G. Dhatt. Modélisation des Structures par Eléments Finis. Vol.3: Coques. Hermes, Paris, 1992.

[6] Kai-Uwe Bletzinger and Ekkehard Ramm. Structural optimization and form finding of light weight structures. Computers \& Structures, 79(2225):2053 - 2062, 2001. 
[7] D. Chapelle and K.J. Bathe. The Finite Element Analysis of Shells Fundamentals. Springer, second edition, 2011.

[8] Mathieu Durand, Frederic Hauville, Patric Bot, Benoit Augier, Yann Roux, Alban Leroyer, and Michel Visonneau. Unsteady numerical simulations of downwind sails. In InnovSail10, pages 57-63, Lorient, France, July 2010. The Royal Institution of Naval Architects.

[9] Kai Graf and Hannes Renzsch. RANSE investigations of downwind sails and integration into sailing yacht design processes. In The Third High Performance Yacht Design Conference, Auckland, New Zealand, December 2008. The Royal Institution of Naval Architects.

[10] Peter Heppel. Accuracy in sail simulation: Wrinkling and growing fast sails. In High Performance Yacht Design Conference, Auckland, New Zealand, 2002.

[11] Seokwoo Kang and Seyoung Im. Finite element analysis of dynamic response of wrinkling membranes. Computer Methods in Applied Mechanics and Engineering, 173(1-2):227 - 240, 1999.

[12] Jin-Jun Li and Siu-Lai Chan. An integrated analysis of membrane structures with flexible supporting frames. Finite Elem. Anal. Des., 40:529 540, March 2004.

[13] M. Lombardi, M. Cremonesi, A. Giampieri, N. Parolini, and A. Quarteroni. A strongly coupled fluid-structure interaction model for wind-sail simulation. In Proceedings of the 4th High Performance Yacht Design conference, Auckland, 2012.

[14] M. Lombardi, N. Parolini, A. Quarteroni, and G. Rozza. Numerical simulation of sailing boats: Dynamics, FSI, and shape optimization. In Variational Analysis and Aerospace Engineering: Mathematical Challenges for Aerospace Design, volume 66 of Optimization and Its Applications, pages 339-377. Springer, 2012.

[15] K. Lu, M. Accorsi, and J. Leonard. Finite element analysis of membrane wrinkling. International Journal for Numerical Methods in Engineering, 50(5):1017-1038, 2001. 
[16] Sabrina Malpede and Andrea Baraldi. A fully integrated method for optimizing fiber-membrane sails. In The Third High Performance Yacht Design Conference, Auckland, New Zealand, December 2008. The Royal Institution of Naval Architects.

[17] N. Nguyen-Thanh, J. Kiendl, H. Nguyen-Xuan, R. Wüchner, K.U. Bletzinger, Y. Bazilevs, and T. Rabczuk. Rotation free isogeometric thin shell analysis using pht-splines. Computer Methods in Applied Mechanics and Engineering, 200(47-48):3410-3424, 2011.

[18] J.T. Oden and T. Sato. Finite strains and displacements of elastic membranes by the finite element method. International Journal of Solids and Structures, 3(4):471 - 488, 1967.

[19] N. Parolini and A. Quarteroni. Mathematical models and numerical simulations for the America's Cup. Computer Methods in Applied Mechanics and Engineering, 194(9-11):1001-1026, 2005.

[20] P. Prukl. The CST - An insidious survivor from the infancy of FEA. In 11th Symposium on Finite Element Methods in South Africa, Cape town, South Africa, January 1992.

[21] Hannes Renzsch and Kai Graf. Fluid structure interaction simulation of spinnakers - getting closer to reality. In Proceedings of the Second International Conference in High Performance Sailing Yachts, Lorient, France, 2010. The Royal Institution of Naval Architects.

[22] Yann Roux, Mathieu Durand, Alban Leroyer, Patrick Queutey, Michel Visonneau, Jeremie Raymond, Jean-Marie Finot, Frederic Hauville, and Aji Purwanto. Strongly coupled VPP and RANSE code for sailing yacht performance prediction. In The Third High Performance Yacht Design Conference, Auckland, New Zealand, December 2008. The Royal Institution of Naval Architects.

[23] B. Tabarrok and Z. Qin. Nonlinear analysis of tension structures. Computers \& Structures, 45(5-6):973 - 984, 1992.

[24] Alexander Tessler, David Sleight, and John Wang. Nonlinear shell modelling of thin membranes with emphasis on structural wrinkling. Technical Report 1931, American Institute of Aeronautics and Astronautics, 2003. 
[25] Daniele Trimarchi. Analysis of downwind sail structures using non-linear shells finite elements - wrinkle development and fluid interaction effects. $\mathrm{PhD}$ thesis, University of Southampton, 2012.

[26] Daniele Trimarchi, Stephen R. Turnock, Dominique Chapelle, and Dominic Taunton. The use of shell elements to capture sail wrinkles, and their influence on aerodynamic loads. In InnovSail10, Lorient, France, July 2010. The Royal Institution of Naval Architects.

[27] I.M. Viola and R.G.J. Flay. Sail pressures from full-scale, wind-tunnel and numerical investigations. Ocean Engineering, 38(16):1733-1743, 2011.

[28] C.G. Wang, H.F. Tan, X.W. Du, and Z.M. Wan. Wrinkling prediction of rectangular shell-membrane under transverse in-plane displacement. International Journal of Solids and Structures, 44(20):6507 - 6516, 2007.

[29] Y. Wong and Sergio Pellegrino. Wrinkled membranes: Experiments (Part I), analytical models (Part II) and numerical simulations (Part III). Journal of Materials and Structures, 1, 2006.

[30] O.C. Zienkiewicz, R.L. Taylor, and J.Z. Zhu. The Finite Element Method: its Basis and Fundamentals. The Finite Element Method. Elsevier Butterworth-Heinemann, 2005. 